\title{
New Banach space properties of the disc algebra and $H^{\infty}$
}

by

\section{J. BOURGAIN}

Vrije Universiteit, Brussels, Belgium

\section{Introduction}

The purpose of this paper is to prove some new linear properties of the disc algebra $A$ and the space $H^{\infty}$ of bounded analytic functions on the disc. More precisely, results on absolutely summing operators, cotype, finite rank projections and certain sequence properties, such as Dunford-Pettis property and weakly completeness, are obtained.

The main motivation for this work were A. Pelczynski's notes (see [44]), which contain also most of the required prerequisites. Our work extends [44], since it solves several of the main problems. It is also of interest in connection with questions raised in [30], [32], [33], [35], [59]. Besides [44], our references for Banach space theory are [36], [37], [38], [47]. Basic facts about $H^{p}$-spaces can be found in [18], [20], [27], [53], [54].

In what follows, we will first describe the frame of the work and recall some definitions. Then we will summarize the several sections of the paper and state the main results. If $u$ is an operator from a space $X$ into a space $Y$ and $0<p<\infty$, we say that $u$ is $p$-absolutely summing provided there is a constant $\lambda$ such that

$$
\sum\left\|u\left(x_{i}\right)\right\|^{p} \leqslant \lambda^{p} \max \left\{\sum\left|\left\langle x_{i}, x^{*}\right\rangle\right|^{p} ; x^{*} \in X^{*},\left\|x^{*}\right\| \leqslant 1\right\}
$$

holds for all finite sequences $\left(x_{i}\right)$ of elements of $X$. The $p$-summing norm $\pi_{p}(u)$ of $u$ is the smallest $\lambda$ with above property. Let $\Pi_{p}(X, Y)$ be the space of $p$-summing operators from $X$ into $Y$.

For $0<p<1$, the spaces $\Pi_{p}(X, Y)$ coincide and will also be denoted by $\Pi_{0}(X, Y)$, the 0 -summing operators from $X$ into $Y$. Say that $u$ is $p$-integral, resp. strictly $p$-integral, provided $u$ admits a factorization

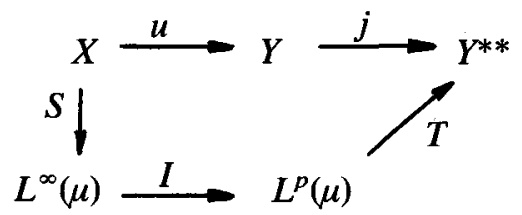

resp.

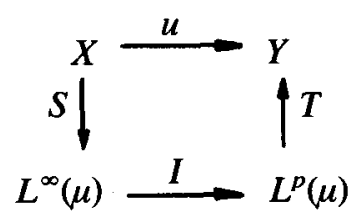

1-848288 Acta Mathematica 152. Imprimé le 17 Avril 1984 
where $\mu$ is a probability measure, $I$ the identity map and $j$ the canonical embedding. The space of strictly $p$-integral operators is denoted by $I_{p}(X, Y)$ and is equipped with the (strictly integral) norm

$$
i_{p}(u)=\inf \|S\|\|T\|
$$

where the infimum is taken over all factorizations. Say that $X$ has Grothendieck property provided any operator from $X$ into $l^{2}$ is 1-summing, thus $B\left(X, l^{2}\right)=\Pi_{1}\left(X, l^{2}\right)$. An equivalent formulation is the equality $B\left(X^{*}, l^{1}\right)=\Pi_{2}\left(X^{*}, l^{1}\right)$. Grothendieck's theorem asserts that $L^{1}(\mu)$-spaces have Grothendieck property. As pointed out in [44] (Theorem 3.2), this general result follows easily from the fact that the operator

$$
H^{\infty} \stackrel{I}{\rightarrow} H^{1} \stackrel{P}{\rightarrow} l^{2}
$$

where $P$ is a Paley projection, is onto. This shows the usefulness of certain specific operators arising in harmonic analysis to the general theory. It is shown in [41] (Theorem 94) that Grothendieck's theorem can be improved to the equality $B\left(l^{1}, l^{2}\right)=\Pi_{0}\left(l^{1}, l^{2}\right)$. A way of seeing this (cf. [32], section 2) is to consider the set

$$
\Lambda=\mathbf{Z}_{+} \cup\left\{-2^{n} ; \quad n=0,1,2, \ldots\right\}
$$

and the orthogonal projection

$$
Q: C_{\Lambda} \rightarrow L_{\left\{-2^{n}\right\}}^{2}
$$

which is again onto by Paley's theorem. Now, for $p>0$, one has the inequality

$$
c_{p}\left(\sum\left|a_{n}\right|^{2}\right)^{1 / 2} \leqslant \inf _{f \in A}\left\{\int_{0}^{2 \pi}\left|\sum_{n=1}^{\infty} a_{n} e^{-i 2^{n} \theta}+f(\theta)\right|^{p} d \theta\right\}^{1 / p}
$$

from which it follows that $Q$ is $p$-summing.

Absolutely summing operators on $A$ appear in the study of certain multipliers. For instance, Paley's theorem that each $\left(A, l^{1}\right)$-multiplier $M$ is $l^{2}$-summable is equivalent to the statement $M \in \Pi_{2}\left(A, l^{1}\right)$. In this spirit, the reader is referred to [35] for a study of translation-invariant absolutely summing operators. Our work actually shows that these results extend to arbitrary operators and that the equality $B\left(A, l^{1}\right)=\Pi_{2}\left(A, l^{1}\right)$ holds in general.

One of the striking facts about operators on the disc algebra is the following extension of the coincidence of the notions of $p$-summing and $p$-integral operators on $C(K)$-spaces (see [44], section 2). 
PROPOSITION 0.1. For $1<p<\infty$, any p-summing operator $u$ on $A$ is strictly $p$ integral. Furthermore

$$
i_{p}(u) \leqslant \text { const. } \frac{p^{2}}{p-1} \pi_{p}(u)
$$

Proposition 0.1 extends the $L^{p}$-boundedness of the Riesz-projection for $1<p<\infty$. It provides a linear invariant which allows for instance to establish the non-isomorphism of $A$ and the polydiscalgebra's. A new proof of Proposition 0.1 based on weighted norm inequalities can be found in [32] (section 2).

Denote $m$ the normalized Haar measure on the circle $\Pi$. If $A$ is a measurable subset of $\Pi$, we shall sometimes use the notation $|A|$ for $m(A)$. If $f \in L^{1}(\Pi), \int f$ means always $\int f d m$. If $H_{0}^{1}$ is the space of integrable functions $f$ on $\Pi$ such that

$$
\hat{f}(n)=\int f(\theta) e^{-i n \theta} m(d \theta)=0 \text { for } n \leqslant 0
$$

then the duality

$$
\langle f, \varphi\rangle=\int f \cdot \varphi d m
$$

identifies $H^{\infty}$ to the dual of the quotient space $L^{1} / H_{0}^{1}$. We consider the quotient map $q: L^{1} \rightarrow L^{1} / H_{0}^{1}$. This map has several remarkable properties which the reader can find in [44] (sections 8 and 9). To each $x$ in $L^{1} / H_{0}^{1}$ corresponds a unique $f$ in $L^{1}$ such that $q(f)=x$ and $\|f\|=\|x\|$. This fact defines the minimum norm lifting $\sigma: L^{1} / H_{0}^{1} \leadsto L^{1}$.

If $A$ is a weakly conditionally compact (WCC) subset of $L^{1} / L_{0}^{1}$, then $\sigma(A)$ is relatively weakly compact in $L^{1}$. Recall that $A$ is WCC provided each sequence in $A$ has a weakly Cauchy sequence. This fact combined with the F. and M. Riesz characterization of $A^{*}$ as

$$
A^{*}=L^{1} / H_{0}^{1} \oplus M_{s}(\Pi) \quad\left(M_{s}=\text { singular measures }\right)
$$

implies that $A^{*}$ is weakly complete and satisfies the Dunford-Pettis property (DPP). It was unknown whether or not $A$ could be replaced by $H^{\infty}$. We answer this affirmatively, by showing that any ultra-power $\left(L^{1} / H_{0}^{1}\right)_{\vartheta \ell}$ of $L^{1} / H_{0}^{1}$ is weakly complete and has DPP. Achieving this requires a local version of the regularity property of $\sigma$ with respect to WCC-sets. This localization, previously sketched in [7], turns out to generalize $\mathbf{J}$. Garnett's theorem that harmonically interpolating sequences in the disc are interpolat- 
ing (see [21]). We will use here a reverse approach (see also the remarks in last section) deriving the lifting theorem from certain facts on interpolating sequences which are apparently new. These will be obtained by dualization of certain results on vectorvalued $H^{1}$-spaces, which are of independent interest.

The fact that the Paley projection $P: A \rightarrow l^{2}$ does not factor through an $L^{1}(\mu)$-space, implies, by general results, that $A$ has no local unconditional structure (see [44], section 4). This means that $A$ cannot be obtained as closure of an increasing sequence $E_{\alpha}$ of finite dimensional subspaces so that $\sup _{\alpha} \operatorname{unc}\left(E_{\alpha}\right)<\infty$, where

$$
\text { unc } X=\inf \left\{\text { unc }\left\{x_{i}\right\} ;\left\{x_{i}\right\} \text { is a basis for } X\right\}
$$

and

$$
\text { unc }\left\{x_{i}\right\}=\sup \left\{\left\|\sum_{i} \pm a_{i} x_{i}\right\| ;\left\|\sum_{i} a_{i} x_{i}\right\|=1\right\} \text {. }
$$

In particular, $A$ is not an $\mathscr{L}^{\infty}$-space (see [36] p. 198 for defirition). However, as we prove, $c_{0}$ is the only (infinite dimensional) complemented subspace of $A$ possessing an unconditional basis and $A$ only admit $c_{0}$-unconditional decompositions (cfr. [57], [58]).

Say that $X$ is a $P_{\lambda}$-space $(\lambda \geqslant 1)$ provided $X$ embeds as $\lambda$-complemented subspace of a $C(K)^{* *}$-space. The structure of finite dimensional $P_{\lambda}$-spaces is not yet understood, except in the case $\lambda$ is close to 1 (see [60]).

We investigate here finite rank projections in $A$ and show that the range has to contain $l_{m}^{\infty}$-spaces of proportional dimension. Besides, any $n$-dimensional $\alpha$-complemented subspace of $A$ is a $P_{\lambda}$ for $\lambda$ of the order $\alpha \cdot \log n$. Natural examples, such as the polynomial spaces $L_{\{0,1, \ldots, n\}}^{\infty}$ show that this result is best possible.

The results on the disc algebra presented in this paper use heavily the fact that $A$ is a log modular algebra. For some of them, also the weak-type property of the Hilbert transform is involved. At this time, we don't know of extensions to other natural spaces, such as the polydisc- and ball-algebra's or spaces defined by singular integrals.

Let us now outline how the remainder of the paper is organized and indicate the main results obtained in the different sections.

In the next section, we derive some simple consequences of the weak-type property of the Hilbert transform. We then apply the classical construction of outer functions to obtain $H^{\infty}$ functions satisfying certain prescribed conditions. More precisely, a Havin type lemma is obtained and certain "truncation" results. The main result is contained in Proposition 1.7, which will be used several times in the paper. 
Section 2 is devoted to the study of absolutely summing operators on the disc algebra and $H^{\infty}$. The central theorem can be stated concretely as follows

THEOREM 0.2. For each finite sequence $\left(x_{k}\right)_{1 \leqslant k \leqslant n}$ in $L^{1} / H_{0}^{1}$, there exists a lifting $\left(f_{k}\right)_{1 \leqslant k \leqslant n}$ in $L^{1}(\Pi)$, i.e. $q\left(f_{k}\right)=x_{k}$ for $k=1, \ldots, n$, such that

$$
\sup _{\varepsilon_{k}= \pm 1}\left\|\sum \varepsilon_{k} f_{k}\right\|_{1} \leqslant C \sup _{\varepsilon_{k}= \pm 1}\left\|\sum \varepsilon_{k} x_{k}\right\|
$$

where $C$ is a fixed constant.

Theorem 0.2 is equivalent to the Grothendieck property of $L^{1} / H_{0}^{1}$. Two different proofs of this fact are presented. The first is the so-called extrapolation-method, which relies on an interpolation enequality for the $p$-summing norms of an operator on $A$. The second, which was suggested in [32], consists in proving that 0-summing operators on $A$ are nuclear. Both approaches have several further consequences for the local structure of the disc algebra.

In section 3, certain vector-valued $\boldsymbol{H}^{\mathbf{1}}$-spaces are characterized. More precisely, the following result is proved.

THEOREM 0.3. Let $X_{0}\left(\right.$ resp. $\left.X_{1}\right)$ be $\mathbf{C}^{N}$ equipped with a weighted $l^{\infty}$ (resp. $\left.l^{1}\right)$ norm. Then the spaces $H_{X_{0} \cup X_{1}}^{1}$ and $H_{X_{0}}^{1} \cup H_{X_{1}}^{1}$ have equivalent norms (up to a fixed constant).

This fact combined with classical Blaschke product techniques has consequences for interpolating sequences in the unit disc, which will be used in the next section. One could use Theorem 0.3 , and the method to derive it, to develop the real interpolation method for $H^{1}$ (and $H^{p}$ ) spaces taking values in Lorentz spaces. Theorem 0.3 can indeed be rephrased in terms of $K$ - or $J$-functionals (see [2] p. 38, for instance). This further development is however not worked out in the paper since it seems us a bit outside its purpose.

The results of section 3 are used in section 4 to derive the following property of the minimum norm lifting of $L^{1} / H_{0}^{1}$.

THEOREM 0.4. Let $\left(x_{k}\right)_{1 \leqslant k \leqslant n}$ be elements of $L^{1 /} H_{0}^{1}$ and assume $f_{k}=\sigma\left(x_{k}\right)$ satisfy

(i) $\int \max _{k} \lambda_{k}\left|f_{k}\right| \geqslant \delta \sum \lambda_{k}\left\|x_{k}\right\|$ whenever $\lambda_{k} \geqslant 0$. 
Then there are $H^{\infty}$-functions $\left(\varphi_{k}\right)_{1 \leqslant k \leqslant n}$ such that

(ii) $\left\|\sum\left|\varphi_{k}\right|\right\|_{\infty} \leqslant 1$

(iii) $\left|\left\langle f_{k}, \varphi_{k}\right\rangle\right|>\varepsilon(\delta)$ for $k=1, \ldots, n$.

This fact can be seen as a local version of the lifting property of weakly conditionnally compact subsets of $L^{1} / H_{0}^{1}$ by $\sigma$.

In section 5 , further linear properties of $H^{\infty}$ are obtained. We first combine Theorem 0.4 with results of $\mathrm{N}$. Tomczak-Jaegermann [56] to prove that any finite dimensional well-complemented subspace of $H^{\infty}$ contains $l_{d}^{\infty}$ subspaces of proportional dimension. Theorem 0.4 is then used to extend J. Chaumat's results (see [15]) on the Dunford-Pettis property and weakly completeness of $L^{1} / H_{0}^{1}$ to the space $\left(H^{\infty}\right)^{*}$. Our method uses ultraproduct representation, which in this context seems the most convenient form of the local reflexivity principle.

Section 6 contains further extensions and applications. Results of D. Marshall [39] allow to generalize part of our work to closed subalgebra's of $L^{\infty}(\Pi)$ containing $H^{\infty}$. Our results on the Grothendieck property solve affirmatively a question of $N$. Varopoulos on projective tensor algebra's. They also turned out to be useful in a recent construction of Banach spaces in connection with some conjectures of A. Grothendieck on tensor products (see [50]).

Part of the material presented here was already announced in the C.R. Acad. Sci. Paris notes [4] and [6]. The reader will find a summary in [11].

\section{Preliminaries and decomposition lemma}

Let us first fix some notation.

$D=\{z \in \mathbf{C} ;|z|<1\}$ is the open unit disc and $\Pi, m$ the circle equipped with Haar measure. Denote $P_{r}(0 \leqslant r<1)$ the Poisson kernel, $\mathscr{R}=\mathscr{R}_{+}$(resp. $\mathscr{R}_{-}$) the positive (resp. negative) Riesz projection and $\mathscr{H}$ the Hilbert transform.

Define for convenience

$$
\begin{gathered}
\|f\|_{W}=\sup _{\lambda>0} \lambda m[|f|>\lambda] \quad \text { for } f \text { measurable on } \Pi \\
\|f\|_{*}=\|f\|_{L^{1} / H_{0}^{1}}=\inf \left\{\|f+h\|_{1}, h \in H_{0}^{1}\right\} \text { for } f \in L^{1}(\Pi) .
\end{gathered}
$$

The restriction map $\left.f \mapsto f\right|_{\Pi}$ gives an isometric embedding of $A$ in $C(\Pi)$. 
Identifying the $H^{p}$-function with its radial limit, the space $H^{p}$ can be seen as subspace of $L^{p}(\Pi)$. If it is not specified otherwise, $A$ - and $H^{p}$-functions will always be seen as functions on $\Pi$. Through the paper, $C$ will be some numerical constant.

Proposition 1.1. Assume $\mu$ in M(I). Then

(i) For $\alpha<1, \mathscr{R}\left(\mu * P_{r}\right)$ converges in $L^{\alpha}(\Pi)$ for $r \rightarrow 1$.

(ii) $\|f\|_{W} \leqslant C\|\mu\|$, where $f=\sup _{r<1}\left|\mathscr{R}\left(\mu * P_{r}\right)\right|$.

The reader will find a detailed exposition of these classical facts in [20] (see Theorem 3.1 p. 57 , Theorem 2.1 p. 111).

LEMMA 1.2. If $\omega \in L_{+}^{\infty}(\Pi)$ and $0 \leqslant \alpha<1$, then

$$
\int|f|^{\alpha} \omega \leqslant \frac{2}{1-\alpha}\|\omega\|_{1}^{1-\alpha}\|\omega\|_{\infty}^{\alpha}\|f\|_{W}^{\alpha}
$$

Proof. Define $\Delta(\lambda)=m[|f|>\lambda]$ and fix $0<\lambda_{0}<\infty$. Then, by partial integration

$$
\int|f|^{\alpha} \omega \leqslant \lambda_{0}^{\alpha}\|\omega\|_{1}+\alpha\left\{\int_{0}^{\infty} \Delta\left(\lambda \vee \lambda_{0}\right) \lambda^{\alpha-1} d \lambda\right\}\|\omega\|_{\infty} \leqslant \lambda_{0}^{\alpha}\|\omega\|_{1}+\frac{1}{1-\alpha} \lambda_{0}^{\alpha-1}\|f\|_{W}\|\omega\|_{\infty} .
$$

Taking then

$$
\lambda_{0}=\frac{\|\omega\|_{\infty}\|f\|_{W}}{\|\omega\|_{1}}
$$

the required inequality follows.

As consequence of Proposition 1.1 and Lemma 1.2, we get

LEMMA 1.3. If $f \in L^{1}(\Pi), \omega \in L_{+}^{\infty}(\Pi)$ and $0 \leqslant \alpha<1$, then

$$
\int\left|\mathscr{R}_{-}(f)\right|^{\alpha} \omega \leqslant \frac{C}{1-\alpha}\|\omega\|_{1}^{1-\alpha}\|\omega\|_{\infty}^{\alpha}\|f\|_{*}^{a}
$$

Let us recall the construction of an outer function. Assume $f>0$ a bounded measurable function on $\Pi$ and $\log f$ in $L^{1}(\Pi)$. If for $z \in D$, we define

$$
g(z)=\exp \left\{\int \log f(\theta) \frac{e^{i \theta}+z}{e^{i \theta}-z} m(d \theta)\right\}
$$

then $g$ is an $H^{\infty}$-function and has boundary value $f e^{i \mathscr{K}(\log f)}$. 
Next lemma is related to the so-called Havin lemma (see [25]).

LEMMA 1.4. If $A$ is a measurable subset of $\Pi$ and $0<\varepsilon<1$, then there are $H^{\infty}$ functions $\varphi$ and $\psi$ such that

(i) $|\varphi|+|\psi| \leqslant 1$

(ii) $|\varphi(z)-1 / 5| \leqslant \varepsilon$ for $z \in A$

(iii) $|\psi(z)| \leqslant \varepsilon$ for $z \in A$

(iv) $\|\varphi\|_{1} \leqslant C\left(\log \varepsilon^{-1}\right)^{2}|A|$

(v) $\|1-\psi\|_{2} \leqslant C\left(\log \varepsilon^{-1}\right)|A|^{1 / 2}$.

Proof. Only the $L^{2}$-boundedness of the Hilbert transform is involved here. Take first

$$
\tau=1-(1-\varepsilon) \chi_{A}
$$

and consider the $H^{\infty}$-function

$$
f=\tau e^{i \mathscr{H}(\log \tau)}
$$

Then $|f|=\varepsilon$ on $A$ and since

$$
\begin{aligned}
|1-f| & \leqslant|1-\tau|+\left\{(1-\cos \mathscr{H}(\log \tau))^{2}+\sin ^{2} \mathscr{H}(\log \tau)\right\}^{1 / 2} \\
& \leqslant|1-\tau|+|\mathscr{H}(\log \tau)|
\end{aligned}
$$

we get

$$
\|1-f\|_{2} \leqslant\|1-\tau\|_{2}+\|\log \tau\|_{2} \leqslant\left(1+\log \frac{1}{\dot{\varepsilon}}\right)|A|^{1 / 2}
$$

Thus if $\varphi=\frac{1}{5}(1-f)^{2}$, (ii) and (iv) are fulfilled. Take now

$$
\varkappa=1-|\varphi| \text { and } g=x e^{i \mathscr{K}(\log x)} .
$$

Then $|\varphi|+|g| \leqslant 1$ and one verifies easily that $\|1-g\|_{2} \leqslant C(\log 1 / \varepsilon)|A|^{1 / 2}$.

Define $\psi=f \cdot g$, which satisfies clearly (i), (iii) and (v).

LEMMA 1.5. There is a constant $C$ such that if $f$ is positive measurable on $\Pi$ and $0<\lambda<\infty$, one can find $\varphi \in H^{\infty}$ satisfying

(i) $\|\varphi\|_{\infty} \leqslant 3$

(ii) $|\varphi| f \leqslant 3 \lambda$

(iii) $\|1-\varphi\|_{1} \leqslant C \lambda^{-1}\|f\|_{W}$

(iv) $\|(1-\varphi) f\|_{1} \leqslant C \int_{[f>\lambda]} f$. 
Proof. If $\|f\|_{W}=\infty$, there is nothing to prove. Otherwise, the function

$$
\tau=\left[\max \left(\lambda^{-1} f, 1\right)\right]^{-1}
$$

has integrable logarithm and one can consider the $H^{\infty}$-function

$$
\varphi_{1}=\tau e^{i \mathscr{K}(\log \tau)} .
$$

Define $\varphi=1-\left(1-\varphi_{1}\right)^{2}=2 \varphi_{1}-\varphi_{1}^{2}$ for which $|\varphi| \leqslant 3\left|\varphi_{1}\right|$. Hence (on $\Pi$ )

$$
|\varphi| f \leqslant 3 \tau f \leqslant 3 \lambda .
$$

Also

$$
\|1-\varphi\|_{1}=\int\left|1-\varphi_{1}\right|^{2} \leqslant 2 \int(1-\tau)^{2}+2 \int|\mathscr{H}(\log \tau)|^{2}
$$

where

$$
\int(1-\tau)^{2} \leqslant m[f>\lambda]
$$

and

$$
\int|\mathscr{H}(\log \tau)|^{2} \leqslant \int|\log \tau|^{2}=\int_{[f>\lambda]}\left(\log \frac{f}{\lambda}\right)^{2} \leqslant C \lambda^{-1}\|f\|_{W}
$$

Because $(\log x)^{2} \leqslant x$ for $x>1$, it follows that

$$
\|1-\varphi\|_{1} \leqslant C \lambda^{-1}\|f\|_{W} \text { and }\|1-\varphi\|_{1} \leqslant C \lambda^{-1} \int_{[\gamma>\lambda]} f .
$$

Finally

$$
\|(1-\varphi) f\|_{1} \leqslant 4 \int_{[f>\lambda]} f+\lambda\|1-\varphi\|_{1}
$$

implying (iv).

As a consequence of preceding lemma, we get following Marcinkiewicz decomposition for $H^{p}$ functions.

PROPOSITION 1.6. For given $0<p<\infty$, there is a constant $C_{p}$ such that if $f \in H^{p}$ and $0<\lambda<\infty$, there is a decomposition in $H^{p}$

$$
f=f_{1}+f_{2}
$$


where

(i) $\left|f_{1}\right| \leqslant C_{p}|f|$ and $\left|f_{2}\right| \leqslant C_{p}|f|$

(ii) $\left|f_{1}\right| \leqslant C_{p} \lambda$

(iii) $\int\left|f_{2}\right|^{p} \leqslant C_{p} \int_{[|f|>\lambda]}|f|^{p}$.

Proof. Apply preceding result to the $L^{1}$-function $\mid f^{p}$ replacing $\lambda$ by $\lambda^{p}$. Let $\varphi$ be the $H^{\infty}$-function obtained in this way. If $p \geqslant 1$, define

$$
f_{1}=f \varphi \text { and } f_{2}=f(1-\varphi)
$$

For $0<p<1$, define first

$$
\varphi_{1}=1-\left(1-\varphi^{k}\right)^{k} \quad \text { where } k=\left[\frac{1}{p}\right]+1
$$

and take again $f_{1}=f \varphi_{1}, f_{2}=f\left(1-\varphi_{1}\right)$.

In what follows, crucial use will be made of the following result.

Proposition 1.7. There is a constant $C$ such that given a positive $L^{1}(\Pi)$ function $f, \int f=1$, and $0<\delta<1$, there are positive scalars $\left(c_{i}\right)$ and sequences $\left(\theta_{i}\right),\left(\tau_{i}\right)$ of $H^{\infty}$ functions satisfying following conditions

(i) $\left\|\theta_{i}\right\|_{\infty} \leqslant C$

(ii) $\Sigma\left|\tau_{i}\right| \leqslant C$

(iii) $\left|\tau_{i}\right| f \leqslant c_{i}$

(iv) $\Sigma c_{i}\left\|\tau_{i}\right\|_{1} \leqslant C \delta^{-C}$

(v) $\int\left|1-\sum \theta_{i} \tau_{i}^{2}\right| f \leqslant \delta$

Proof. Fix a positive number $M=M(\delta)>1$ which will be specified later and define for $i \in \mathbf{Z}$

$$
A_{i}=\left[M^{i} \leqslant f<M^{i+1}\right]
$$

Clearly $\left|A_{i}\right| \leqslant M^{-i} \int_{A_{i}} f \leqslant M^{-i}$. Apply Lemma 1.4 to each set $A_{i}$, taking $\varepsilon=M^{-1}$, which leads to $H^{\infty}$-functions $\varphi_{i}, \psi_{i}$. Hence, by (i), (v) of Lemma 1.4,

$$
\left|\psi_{i+s}^{s}-1\right| \leqslant s\left|\psi_{i+s}-1\right| \quad \text { because }\left|\psi_{i+s}\right| \leqslant 1 \text { for } s=1,2, \ldots
$$


and thus

$$
\sum_{s=1}^{\infty}\left\|1-\psi_{i+s}^{s}\right\|_{1} \leqslant \sum_{s=1}^{\infty} s\left\|1-\psi_{i+s}\right\|_{2} \leqslant C \log M \sum_{s=1}^{\infty} s\left|A_{i+s}\right|^{1 / 2}<\infty .
$$

Therefore, the formula

$$
\tau_{i}=5 \varphi_{i} \prod_{s=8}^{\infty} \psi_{i+s}^{s}
$$

defines an $H^{\infty}$-function. Moreover, by (ii) and (v) of Lemma 1.4, and the CauchySchwartz inequality

$$
\text { (vi) } \begin{aligned}
\sum_{i} \int_{A_{i}}\left|1-\tau_{i}\right| f & \leqslant \sum_{i} \int_{A_{i}}\left|1-5 \varphi_{i}\right| f+\sum_{i} \sum_{s \geqslant 8} s \int_{A_{i}}\left|1-\psi_{i+s}\right| f \\
& \leqslant 5 \varepsilon+C \log M \sum_{i} M^{i+1} \sum_{s \geqslant 8} s\left|A_{i}\right|^{1 / 2}\left|A_{i+s}\right|^{1 / 2} \\
& \leqslant 5 \varepsilon+C \log M \sum_{s \geqslant 8} s M^{1-s / 2} \sum_{i}\left(M^{i}\left|A_{i}\right|\right)^{1 / 2}\left(M^{i+s}\left|A_{i+s}\right|\right)^{1 / 2} \\
& \leqslant 5 M^{-1}+C \log M \sum_{s \geqslant 8} s M^{1-s / 2}=C M^{-1}
\end{aligned}
$$

Further

(vii) $f\left|\tau_{i}\right| \leqslant 5 M^{i+8}$

since by Lemma 1.4(iii) we get on the set $A_{j}$ for $j \geqslant i+8$

$$
f\left|\tau_{i}\right| \leqslant 5 M^{j+1}\left|\psi_{j}\right|^{j-i} \leqslant 5 M^{j+1} \varepsilon^{j-i}=5 M^{i+1}
$$

while $f\left|\tau_{i}\right| \leqslant 5 f \leqslant 5 M^{i+8}$ on $\cup_{j<i+8} A_{j}$.

Also, by Lemma 1.4(iv)

(viii) $\quad \sum M^{i}\left\|\tau_{i}\right\|_{1}=5 \sum M^{i}\left\|\varphi_{i}\right\|_{1} \leqslant C(\log M)^{2} \sum M^{i}\left|A_{i}\right| \leqslant C(\log M)^{2}$.

For $t=0,1,2, \ldots, 7$, write for convenience $i \equiv t$ provided $i=t(\bmod 8)$. 
Iteration of Lemma 1.4(i) shows then that

(ix)

$$
\sum_{i=i}\left|\tau_{i}\right| \leqslant 5 \sum_{i=i}\left|\varphi_{i}\right| \prod_{\substack{j=1 \\ j>i}}\left|\psi_{j}\right| \leqslant 5
$$

Define for each $t$

$$
B_{t}=\bigcup_{i=t} A_{i} \quad \text { and } \quad \eta_{t}=\sum_{i \equiv t} \tau_{i}^{2}
$$

Thus

(x) $\left\|\eta_{\imath}\right\|_{\infty} \leqslant 25$.

From Lemma 1.4(iii) and (iv), we get clearly

$$
\begin{aligned}
\int_{B_{t}}\left|1-\eta_{t}\right| f & \leqslant \sum_{i \cong t}\left\{\int_{A_{i}}\left|1-\tau_{i}^{2}\right| f+\sum_{\substack{j=t \\
j \neq i}} \int_{A_{i}}\left|\tau_{j}\right|^{2} f\right\} \\
& \leqslant 6 \sum_{i \cong t} \int_{A_{i}}\left|1-\tau_{i}\right| f+25 \sum_{i \equiv t} M^{i+1}\left\{\sum_{j \leqslant i-8} \int_{A_{i}}\left|\psi_{i}\right|^{i-j}+\sum_{j \geqslant i+8}\left\|\varphi_{j}\right\|_{1}\right\} \\
& \leqslant C \sum_{i=t} \int_{A_{i}}\left|1-\tau_{i}\right| f+C M^{-6} \sum_{i=t} M^{i}\left|A_{i}\right|+C(\log M)^{2} \sum_{i \equiv t} M^{i+1} \sum_{j \geqslant i+8}\left|A_{j}\right|
\end{aligned}
$$

Hence, by (vi)

(xi)

$$
\sum_{t} \int_{B_{t}}\left|1-\eta_{t}\right| f \leqslant C M^{-1}+C M^{-6}+C(\log M)^{2} M^{-6}=C M^{-1}
$$

Define the $H^{\infty}$-functions $\theta_{i}$ as follows

$$
\left\{\begin{array}{l}
\theta_{i}=\left(1-\eta_{t+1}\right)\left(1-\eta_{t+2}\right) \ldots\left(1-\eta_{7}\right) \text { if } i \equiv t<7 \\
\theta_{i}=1 \text { if } i \equiv 7
\end{array}\right.
$$

Consequently

$$
\sum \theta_{i} \tau_{i}^{2}=\sum_{t=0}^{7} \eta_{t} \prod_{u>t}\left(1-\eta_{u}\right)
$$

and therefore

$$
1-\sum \theta_{i} \tau_{i}^{2}=\prod_{t=0}^{7}\left(1-\eta_{t}\right)
$$


By (ix)

(xii) $\Sigma\left|\tau_{i}\right| \leqslant 40$

and from definition and $(\mathrm{x})$

(xiii) $\left\|\theta_{i}\right\|_{\infty} \leqslant 26^{7}$.

Since

$$
\int\left|1-\sum \theta_{i} \tau_{i}^{2}\right| f=\int \prod_{t}\left|1-\eta_{t}\right| f \leqslant C \sum_{t=0}^{7} \int_{B_{t}}\left|1-\eta_{t}\right| f .
$$

(xi) shows that (v) will be satisfied for $M \sim \delta^{-1}$.

Taking $c_{i}=5 M^{i+8}$, conditions (iii) and (iv) follow from (vii) and (viii).

So this completes the proof.

\section{Absolutely summing operators and the cotype property}

For completeness sake, we recall the following fact (cf. [44], Theorem 2.3) concerning the decomposition of an absolutely summing operator $T$ on the disc algebra $A$.

Proposition 2.1. Assume T p-summing ( $p \leqslant 1$ ) on A. Then $T$ has a decomposition $T=T_{1}+T_{2}$, where the components $T_{1}, T_{2}$ fulfil the following conditions:

(i) $\pi_{p}\left(T_{1}\right)^{p}+\pi_{p}\left(T_{2}\right)^{p} \leqslant \pi_{p}(T)^{p}$.

(ii) $\pi_{p}\left(T_{1}\right)$ is realized by a Pietch measure on the circle $\Pi$ belonging to $L^{1}(m)$. Moreover

$$
\pi_{q}\left(T_{1}\right) \leqslant \pi_{q}(T) \text { for } 0<q \leqslant \infty .
$$

(iii) There is a sequence of operators $S_{n}: C(\Pi) \rightarrow A$, such that $\left(T_{2} S_{n}\right)$ converges in $\pi_{p}$-norm to an operator $\tilde{T}_{2}$ satisfying $T_{2}=\tilde{T}_{2} j$ where $j: A \rightarrow C(\Pi)$ is the injection.

Proof. Since $p \geqslant 1$, the $A$. Pietch factorisation theorem (cf. [47]) provides a Radon probability measure $\mu$ on $\Pi$ such that

$$
\|T(\varphi)\| \leqslant \pi_{p}(T)\|\varphi\|_{L^{p}(\mu)} \text { for } \varphi \in A .
$$

Let $d \mu=h d m+d \mu_{s}$ be the Lebesgue decomposition and $L=\cup_{n=1}^{\infty} K_{n}, K_{n}$ compact, a $K_{\sigma^{-}}$ subset of $\Pi$ so that $m(L)=0$ and $\mu_{s}(L)=\left\|\mu_{s}\right\|$. 
By classical results from peak-set theory (see [20], p. 203, Lemma 4.5), it is possible to find a sequence $\left(\psi_{k}\right)$ in $A$ satisfying the conditions

(iv) $\left\|\psi_{k}\right\|_{\infty} \leqslant 1$

(v) $\left(\psi_{k}\right)$ converges pointwise to 0 on the set $L$

(vi) $\left(\psi_{k}\right)$ converges to 1 a.e. (with respect to $m$ ).

Since in particular $\left(\psi_{k}\right)$ converges in $L^{p}(\mu)$, one can define

$$
T_{1}(\varphi)=\lim _{k \rightarrow \infty} T\left(\varphi \psi_{k}\right)
$$

and

$$
T_{2}=T-T_{1}
$$

Clearly

$$
\left\|T_{1}(\varphi)\right\| \leqslant \pi_{p}(T)\|\varphi\|_{L^{p_{(h \cdot d m)}}} \text { and }\left\|T_{2}(\varphi)\right\| \leqslant \pi_{p}(T)\|\varphi\|_{L^{\left.p^{\left(d d u_{s}\right.}\right)}}
$$

from where (i).

A similar reasoning actually shows that $\pi_{p}\left(T_{1}\right)$ is realized by an $m$-regular measure. Also, from definition, it follows immediately that

$$
\pi_{q}\left(T_{1}\right) \leqslant \pi_{q}(T) \text { for all } 0<q \leqslant \infty .
$$

Denote for each $n=1,2, \ldots$,

$$
R_{n}: C(\Pi) \rightarrow C\left(K_{n}\right) \text { the restriction operator }
$$

and

$$
E_{n}: C\left(K_{n}\right) \rightarrow A \text { a norm-preserving extension (cf. [44], Theorem 2.1). }
$$

If one defines $S_{n}=E_{n} R_{n}$, it is easily verified that the sequence $\left(T_{2} S_{n}\right)$ converges in $\pi_{p^{-}}$ norm to an extension $\tilde{T}_{2}$ of $T_{2}$.

Remarks. (1) The first component $T_{1}$ can be extended to $H^{\infty}$, defining

$$
\tilde{T}_{1}(\varphi)=\lim _{r \gtrless^{1}} T_{1}\left(\varphi * P_{r}\right) .
$$

It is indeed clear that the limit exists. This extension will be useful in what follows. 
(2) In case $T$ is 0 -summing, one applies Proposition 2.1 with $p=1$. Thus $T_{1}$ and $T_{2}$ are again 0 -summing. Now, for each $n$, the operator $T_{2} S_{n}$ will therefore be nuclear (see [32], Theorem 5) and therefore also $\tilde{T}_{2}$ and $T_{2}$.

Let us now state our first main result.

THEOREM 2.2. Let $p \geqslant 2$ and $Y$ a Banach space such that any bounded operator from $C$ into $Y$ is p-summing, i.e. $B(C, Y)=\Pi_{p}(C, Y)$. Then also $B(A, Y)=\Pi_{p}(A, Y)$.

$\left(\Pi_{p}\right.$ can be replaced by $I_{p}$, taking Proposition 0.1 in account.)

Theorem 2.2 will be obtained from an extrapolation technique, which was already previously used in [43], [34] and G. Pisier's proof that the quotient of $L^{1}$ by a reflexive subspace verifies the Grothendieck theorem [48].

The main ingredient is an interpolation inequality on the $p$-summing norms, which will be presented in the next sub-section.

\subsection{An interpolation inequality}

Our purpose is to show the following fact:

Proposition 2.3. Assume $1<p<\infty$ and T p-summing on A. Let $p<q<\infty$ and $\theta$ such that

$$
\frac{1}{q^{\prime}}=\theta+\frac{1-\theta}{p^{\prime}} \quad\left(p^{\prime}=\frac{p}{p-1}, q^{\prime}=\frac{q}{q-1}\right)
$$

Then for all $0 \leqslant \phi<\theta$, one has an inequality

$$
i_{q}(T) \leqslant C(p, q, \phi)\|T\|^{\phi} \pi_{p}(T)^{1-\phi}
$$

where, more precisely, $C(p, q, \phi)=C(p) /(\theta-\phi)$.

This result turns out to be sharp, as will be clear from a discussion below.

The proof of Proposition 2.3 depends on the following preliminary decomposition property.

LEMMA 2.4. There is a constant $C>1$ such that under the hypothesis of Proposition 2.3 and for given $0<\delta<1$, the operator $T$ has a decomposition $T=I+R$, where

(i) I is strictly q-integral and

$$
i_{q}(I) \leqslant C_{p}(\theta-\phi)^{-1} \delta^{-C(1-\phi) / p}\|T\|^{\phi} \pi_{p}(T)^{1-\phi}
$$


(ii) $\|R\| \leqslant C\|T\|$ and $\pi_{p}(R) \leqslant C \delta^{1 / p} \pi_{p}(T)$.

where $C_{p}$ is the norm of the Riesz-projection regarded as an operator in $L^{p}$.

Proposition 2.3 is then obtained by an iteration procedure. Starting from $R_{0}=T$, consider successive decompositions $R_{k}=I_{k+1}+R_{k+1}$ according to Lemma 2.4.

We see that for $\delta$ small enough

$$
\pi_{p}\left(R_{k}\right) \rightarrow 0 \quad \text { and } \quad i_{q}\left(I_{k}\right) \leqslant C_{p}(\theta-\phi)^{-1} \delta^{-C(1-\phi) / p}\left(C \delta^{(1-\phi) / p}\right)^{k-1}\|T\|^{\phi} \pi_{p}(T)^{1-\phi} .
$$

Specifying $\delta=(2 C)^{-p /(1-\phi)}$ leads to the estimation

$$
i_{q}(T) \leqslant \sum_{k} i_{q}\left(I_{k}\right) \leqslant C_{p}(\theta-\phi)^{-1}(2 C)^{C} 2\|T\|^{\phi} \pi_{p}(T)^{1-\phi}
$$

and thus

$$
i_{q}(T) \leqslant C(p)(\theta-\phi)^{-1}\|T\|^{\phi} \pi_{p}(T)^{1-\phi}
$$

as required.

Proof of Lemma 2.4. First one can identify $T$ with the component $T_{1}$ in decomposition 2.1. Indeed, since $\tilde{T}_{2}$ is defined on $C(\Pi)$, Hölder's inequality yields immediately (cf. [48], p. 75) that

$$
i_{q}\left(T_{2}\right) \leqslant i_{q}\left(\tilde{T}_{2}\right) \leqslant\left\|\tilde{T}_{2}\right\|^{\theta} \pi_{p}\left(\tilde{T}_{2}\right)^{1-\theta} \leqslant 2\|T\|^{\theta} \pi_{p}(T)^{1-\theta}
$$

Thus $T$ extends to $H^{\infty}$ and there exists $f \in L_{+}^{1}(\Pi), \int f=1$, such that

$$
\|T(\varphi)\| \leqslant \pi_{p}(T)\|\varphi\|_{L^{p(f \cdot d m)}} \text { for } \varphi \in H^{\infty} .
$$

Let $\left(c_{i}\right),\left(\theta_{i}\right),\left(\tau_{i}\right)$ be the sequences obtained by application of Proposition 1.7 to the function $f$, taking $\delta$ as in Lemma 2.4. Define

$$
I(\varphi)=T\left(\varphi \cdot \sum \theta_{i} \tau_{i}^{2}\right) \text { and } R=T-I
$$

which makes sense by (i), (ii) of Proposition 1.7. Also $\|R\| \leqslant C\|T\|$. Since

$$
\|R(\varphi)\| \leqslant \pi_{p}(T)\left\{\int|\varphi|^{p}\left|1-\sum \theta_{i} \tau_{i}^{2}\right|^{p} f d m\right\}^{1 / p}
$$


estimation (v) of Proposition 1.7 combined with (i) and (ii) of Proposition 1.7 show that

$$
\pi_{p}(R) \leqslant C \delta^{1 / p} \pi_{p}(T)
$$

Remains to verify (i). Extend $I$ to $C(\Pi)$ by the formula

$$
I(\varphi)=T\left(\sum_{i} \tau_{i} \mathscr{R}_{+}\left(\theta_{i} \tau_{i} \varphi\right)\right)
$$

which clearly makes sense if $\hat{\varphi}$ has finite spectrum. Now

$$
\|I(\varphi)\|=\sup _{F}\left|\int\left(\sum_{i} \tau_{i} \mathscr{R}_{+}\left(\theta_{i} \tau_{i} \varphi\right)\right) F\right|
$$

for $F \in L^{1}(\Pi)$ satisfying

$$
\left|\int \varphi F d m\right| \leqslant\|T(\varphi)\| \quad \text { for all } \varphi \in H^{\infty}
$$

Also

$$
\int\left(\sum \tau_{i} \mathscr{R}_{+}\left(\theta_{i} \tau_{i} \varphi\right)\right) F=\lim _{r \gtrless^{1}} \int \sum \theta_{i} \tau_{i} \varphi \mathscr{R}_{-}^{r}\left(\tau_{i} F\right)
$$

defining for convenience $\mathscr{R}_{-}^{r}=\mathscr{R}_{-} * P_{r}$.

Fixing $r<1$, application of the Hölder inequality leads to

$$
\int \sum\left|\tau_{i}\right||\varphi|\left|\mathscr{R}_{-}^{r}\left(\tau_{i} F\right)\right| \leqslant\left\{\sum \int\left|c_{i}^{-1} \mathscr{R}_{-}^{r}\left(\tau_{i} F\right)\right|^{q^{\prime}} c_{i}\left|\tau_{i}\right|\right\}^{1 / q^{\prime}}\left\{\int|\varphi|^{q}\left(\sum c_{i}\left|\tau_{i}\right|\right)\right\}^{1 / q} .
$$

By definition of $\theta$, we get

$$
q^{\prime}=\left(\frac{\phi}{\alpha} q^{\prime}\right) \alpha+\left(1-\frac{\phi}{\alpha} q^{\prime}\right) p^{\prime} \quad \text { where } \alpha=p\left(p-1+\frac{\theta}{\phi}\right)^{-1} .
$$

So, applying the Hölder inequality for sequences, we get the estimation $U \times V$ on the first factor of the preceding inequality, where

$$
\begin{aligned}
& U=\left\{\sum \int\left|c_{i}^{-1} \mathscr{R}_{-}^{r}\left(\tau_{i} F\right)\right|^{\alpha} c_{i}\left|\tau_{i}\right|\right\}^{\phi / a} \text { and } \\
& V=\left\{\sum \int\left|c_{i}^{-1} \mathscr{R}_{-}^{r}\left(\tau_{i} F\right)\right| p^{\prime} c_{i}\left|\tau_{i}\right|\right\}^{1 / q^{\prime}-\phi / \alpha}
\end{aligned}
$$

2-848288 Acta Mathematica 152. Imprimé le 17 Avril 1984 
18

J. BOURGAIN

To estimate $U$, apply first Lemma 1.3 with $\omega=\left|\tau_{i}\right|$, which gives

$$
\int\left|\mathscr{R}_{-}\left(\tau_{i} F\right)\right|^{\alpha}\left|\tau_{i}\right| \leqslant \frac{C}{1-\alpha}\left\|\tau_{i}\right\|_{1}^{1-\alpha}\left\|\tau_{i} F\right\|_{* \cdot}^{\alpha}
$$

Thus, using (ii) and (iv) of Proposition 1.7

$$
\begin{aligned}
U & \leqslant\left(\frac{C}{1-\alpha}\right)^{\phi / \alpha}\left(\sum c_{i}\left\|\tau_{i}\right\|_{1}\right)^{(1-\alpha) / \alpha) \phi}\left(\sum\left\|\tau_{i} F\right\|_{*}\right)^{\phi} \\
& \leqslant\left(\frac{C}{1-\alpha}\right)^{\phi / \alpha} \delta^{-C \phi(1-\alpha) / \alpha}\|F\|_{*}^{\phi} \\
& \leqslant C \frac{(\theta p)^{\phi}}{(\theta-\phi)^{\theta}} \delta^{-C(\theta-\phi) / p}\|T\|^{\phi} \\
& \leqslant p^{\phi}(\theta-\phi)^{-1} \delta^{-C(1-\phi) / p}\|T\|^{\phi}
\end{aligned}
$$

The $L^{p}-L^{p^{\prime}}$ duality shows that

$$
\left\{\sum \int\left|c_{i}^{-1} \mathscr{R}_{-}\left(\tau_{i} F\right)\right|^{p^{\prime}} c_{i}\left|\tau_{i}\right|\right\}^{\mathrm{u} p^{\prime}}=\sup _{\left(\xi_{i}\right)}\left|\sum \int \zeta_{i} \mathscr{R}_{-}^{r}\left(\tau_{i} F\right)\right| \tau_{i}||
$$

over the sequence $\left(\zeta_{i}\right)$ fulfilling

$$
\sum_{i} \int\left|\zeta_{i}\right|^{p}\left(c_{i}\left|\tau_{i}\right|\right) \leqslant 1
$$

Since

$$
\sum \int \zeta_{i} \mathscr{R}_{-}\left(\tau_{i} F\right)\left|\tau_{i}\right|=\int\left(\sum \tau_{i} \mathscr{R}_{+}^{r}\left(\zeta_{i}\left|\tau_{i}\right|\right)\right) F
$$

we obtain estimation by

$$
\left\|T\left(\sum \tau_{i} \mathscr{R}_{+}\left(\zeta_{i} \mid \tau_{i}\right)\right)\right\| \leqslant \pi_{p}(T)\left\{\int\left|\sum \tau_{i} \mathscr{R}_{+}\left(\zeta_{i} \mid \tau_{i}\right)\right|^{p} f\right\}^{1 / p} .
$$

By Proposition 1.7 (ii), (iii) and the M. Riesz theorem

$$
\begin{aligned}
\int\left|\sum \tau_{i} \mathscr{R}_{+}\left(\zeta_{i} \mid \tau_{i}\right)\right| p & \leqslant C \int \sum\left|\tau_{i}\right|\left|\mathscr{R}_{+}\left(\zeta_{i} \mid \tau_{i}\right)\right|^{p} f \\
& \leqslant C \sum c_{i} \int\left|\mathscr{R}_{+}\left(\zeta_{i}\left|\tau_{i}\right|\right)\right|^{p}
\end{aligned}
$$




$$
\begin{aligned}
& \leqslant C C_{p} \sum c_{i} \int\left|\zeta_{i}\right|^{p}\left|\tau_{i}\right| \\
& \leqslant C C_{p} .
\end{aligned}
$$

Consequently

$$
V \leqslant\left(C C_{p} \pi_{p}(T)\right)^{p^{\prime}\left(1 / q^{\prime}-\phi / a\right)} \leqslant\left(C C_{p} \pi_{p}(T)\right)^{1-\phi}
$$

By completion, $I$ extend to $C(\Pi)$ and

$$
\begin{aligned}
i_{q}(I) & \leqslant U V\left(\sum c_{i}\left\|\tau_{i}\right\|_{1}\right)^{1 / q} \\
& \leqslant U V \delta^{-C(1-\theta) / p} \\
& \leqslant C_{p}(\theta-\phi)^{-1} \delta^{-C(1-\phi) / p}\|T\|^{\phi} \pi_{p}(T)^{1-\phi}
\end{aligned}
$$

as desired.

\subsection{Consequences}

Let us first proceed with the proof of Theorem 2.2. Denote $\gamma_{\infty}(T)$ the factorization constant of the operator $T$ through an $L^{\infty}(\mu)$-space. By Proposition 2.3, we get for $T \in \Pi_{p}(A, Y)$

$$
\gamma_{\infty}(T) \leqslant i_{q}(T) \leqslant \frac{C(p)}{\theta-\phi}\|T\|^{\phi} \pi_{p}(T)^{1-\phi}
$$

for $p<q$ and $\phi<\theta$. Since $\theta \rightarrow 1$ for $q \rightarrow \infty$

$$
\gamma_{\infty}(T) \leqslant \frac{C(p)}{1-\phi}\|T\|^{\phi} \pi_{p}(T)^{1-\phi}
$$

If now $Y$ satisfies the hypothesis $B(C, Y)=\Pi_{p}(C, Y)$, we get also

$$
\pi_{p}(T) \leqslant C(Y) \gamma_{\infty}(T) \text { for a fixed constant } C(Y)
$$

Hence

$$
\pi_{p}(T) \leqslant\left(\frac{C(Y) C(p)}{1-\phi}\right)^{1 / \phi}\|T\|
$$

proving the equivalence of operator- and $p$-summing norms for finite rank operators 
from $A$ into $Y$. Since $A$ has the bounded approximation property (in fact $A$ has a basis [3]), we conclude that $B(A, Y)=\Pi_{p}(A, Y)$.

If we choose in $\left(^{*}\right)$

$$
\phi=1-\left(\log \frac{\pi_{p}(T)}{\|T\|}\right)^{-1}
$$

we find

$$
\gamma_{\infty}(T) \leqslant C(p)\|T\| \log \frac{\pi_{p}(T)}{\|T\|}
$$

Hence

THEOREM 2.5. If $T \in \Pi_{p}(A, Y)$, then $T$ has an extension $\tilde{T}$ to $C(\Pi)$ satisfying

$$
\|\tilde{T}\| \leqslant C(p)\|T\| \log \frac{\pi_{p}(T)}{\|T\|} .
$$

Since always

$$
\pi_{2}(T) \leqslant\|T\|(\operatorname{rank} T)^{1 / 2}
$$

for finite rank operators, the following corollary is immediate:

COROLLARY 2.6. (i) A rank $n$ operator $T$ on the disc algebra has an extension $\tilde{T}$ to $C(\Pi)$ satisfying $\|\bar{T}\| \leqslant C(2)(\log n)\|T\|$.

(ii) If $X$ is an $n$-dimensional subspace of $A$ complemented by a projection $P$, then $X$ is a $P_{\lambda}$-space with $\lambda \leqslant C(2)(\log n)\|P\|$.

This result, answering affirmatively problems raised in [46] and [59], is best possible as we will indicate at the end of this section.

Combining Theorem 2.2 with Grothendieck's fundamental theorem $B\left(C, l^{1}\right)=$ $\Pi_{2}\left(C, l^{1}\right)$ and a result due to Maurey (see [40]), the following consequences are derived.

CoROllaRy 2.7. $B\left(A, l^{1}\right)=\Pi_{2}\left(A, l^{1}\right)$, or equivalently, $A^{*}$ verifies the Grothendieck theorem.

COROLLARY 2.8. If $c_{0}$ is not finitely representable in $Y$, then $B(A, Y)=\Pi_{p}(A, Y)$ for some $p<\infty$. In particular, if $Y$ is a cotype 2 space, then $B(A, Y)=\Pi_{2}(A, Y)$. 
Let us recall that $Y$ has cotype $q(2 \leqslant q<\infty)$ provided

$$
\int\left\|\sum \varepsilon_{i} y_{i}\right\| d \varepsilon \geqslant \gamma\left(\sum\left\|y_{i}\right\|^{q}\right)^{1 / q}
$$

holds for some constant $\gamma>0$ and for all finite sequences $\left(y_{i}\right)$ in $Y$.

(As usually, $\left(\varepsilon_{i}\right)$ denotes the Rademacher sequence.)

Proof of Theorem 0.2. Consider elements $\left(x_{k}\right)_{1 \leqslant k \leqslant n}$ in $L^{1} / H_{0}^{1}$ and the operator $T: A \rightarrow l_{n}^{1}$ given by $T(\varphi)=\left(\left\langle\varphi, x_{k}\right\rangle\right)_{1 \leqslant k \leqslant n}$. Clearly

$$
\|T\| \leqslant 2 \sup _{\varepsilon_{k}= \pm 1}\left\|\sum \varepsilon_{k} x_{k}\right\| .
$$

By the extension property for 2-summing norm and Corollary 2.7 , there exists an operator $\tilde{T}: C(\Pi) \rightarrow l_{n}^{1}$ satisfying $\tilde{T} j=T$, where $j: A \rightarrow C(\Pi)$ is the injection, and $\|\tilde{T}\| \leqslant \pi_{2}(\tilde{T})=\pi_{2}(T) \leqslant C\|T\|$. Denote $\mu_{k} \in M(\Pi)$ the $k$ th component of $(\tilde{T})^{*}$. Then

$$
\sup _{\varepsilon_{k}= \pm 1}\left\|\sum \varepsilon_{k} \mu_{k}\right\| \leqslant\left\|(\tilde{T})^{*}\right\| \leqslant C\|T\| .
$$

Moreover, if for $k=1, \ldots, n$ we consider $\tilde{x}_{k}$ in $L^{1}(\Pi)$ representing $x_{k}$, it follows that $\left(\mu_{k}-\tilde{x}_{k}\right) \perp A$, thus $\mu_{k}-\tilde{x}_{k}$ is in $H_{0}^{1}$ and in particular $\mu_{k} \ll m$.

The following observation, due to Figiel and Pisier (cfr. [19] and the remarks at the end of [58]) is well known. We include its proof for selfcontainedness sake.

PROPOSITION 2.9. Any space $X$ with the Grothendieck property, isomorphic to its direct sum $\left(\sum_{n=1}^{\infty} X\right)_{1}$ in $l^{\prime}$-sense, is of cotype 2.

Proof. If $\Delta$ denotes the Cantor group, then $L_{X}^{1}(\Delta)$ verifies the Grothendieck theorem. For $\xi \in L_{X}^{\prime}(\Delta)$ and $S$ a finite set of positive integers, denote $\hat{\xi}(S)$ the corresponding Fourier-Walsh coefficient of $\xi$. Fix a sequence $\left(x_{i}^{*}\right)$ in $X^{*}$ satisfying

$$
\left(\sum\left|\left\langle x_{i}^{*}, x\right\rangle\right|^{2}\right)^{1 / 2} \leqslant\|x\| \text { for all } x \in X
$$

Then the map $\alpha: L_{X}^{1} \rightarrow l^{2}$ defined by $\alpha(\xi)=\left(\left\langle\xi\{i\}, x_{i}^{*}\right\rangle\right)$ is norm-1 bounded and hence $\pi_{1}(\alpha) \leqslant C(X)$. Given an arbitrary (finite) sequence $\left(x_{i}\right)$ in $X$, it follows thus

$$
C(X) \int\left\|\sum \varepsilon_{i} x_{i}\right\| d \varepsilon \geqslant \sum\left\|\alpha\left(x_{i} \otimes \varepsilon_{i}\right)\right\| \geqslant \sum\left|\left\langle x_{i}, x_{i}^{*}\right\rangle\right| .
$$


Hence, in particular

$$
\int\left\|\sum \varepsilon_{i} x_{i}\right\| d \varepsilon \geqslant C(X)^{-1}\left(\sum\left\|x_{i}\right\|^{2}\right)^{1 / 2}
$$

by an appropriate choice of the $x_{i}^{*}$.

The reader is referred to [57] and [58] for the following facts:

PROPOSITION 2.10. (i) For $1 \leqslant p \leqslant \infty$, the space $H^{p}$ is isomorphic to its direct sum $\left(\sum_{n=1}^{\infty} H^{p}\right)_{p}$.

(ii) The disc algebra $A$ is isomorphic to $\left(\sum_{n=1}^{\infty} A\right)_{c_{0}}$.

Combining Corollary 2.7 and Proposition 2.10 (ii) we get

COROLlaRY 2.11. The dual of the disc algebra $A^{*}$ is a space of cotype 2.

By arguments of local reflexivity, Corollaries 2.7 and 2.11 remain valid if $A$ is replaced by $H^{\infty}$. Since it is not known if $H^{\infty}$ has the bounded approximation property, extension of Corollary 2.8 to $H^{\infty}$ is not clear. However, the result holds assuming that $Y$ has the bounded approximation property.

Next results are formal consequences of the cotype 2 and Grothendieck property.

COROLLARY 2.12. (i) $c_{0}$ is up to isomorphism the only complemented subspace of $A$ possessing an unconditional basis.

(ii) If $\Sigma X_{j}$ is an unconditional decomposition of $A\left(\right.$ resp. $\left.H^{\infty}\right)$, then $\Sigma X_{j}$ is a $c_{0}$ sum (resp. $l^{\infty}$-sum) (cf. Proposition 2.10).

Corollary 2.8 allows to improve Theorem 2 of [30] as follows

COROLLARY 2.13. Given a reflexive subspace $X$ of $A^{*}$, there exists an embedding $\beta: X \rightarrow C(\Pi)^{*}$ such that moreover $j^{*} \beta(x)=x$ for $x \in X$, where $j: A \rightarrow C(\Pi)$ is again the injection.

Proof. By Lemma 3 of [30], a reflexive subspace $X$ of $A^{*}$ does not contain $l_{n}^{1 \text { 's }}$ uniformly and hence $X^{*}$ has a finite cotype. Therefore, by Corollary 2.8, denoting $i: X \rightarrow A^{*}$ the injection, $\left.i^{*}\right|_{A}=T$ is $p$-summing and thus $p$-integral for some $p<\infty$. Thus $T$ factors through an $L^{\infty}(\mu)$-space and can be extended to $C(\Pi)$. Let $\tilde{T}$ be this extension. Since $\tilde{T} j=T$, it follows that $i=j^{*} \beta$ where $\beta$ is the restriction of $(\tilde{T})^{*}$ to $X$.

Further results concerning projections in the spaces $L^{1 /} H_{0}^{1} A$ and $H^{\infty}$ will be presented in section 5 . 


\subsection{An alternative approach}

If $X$ is a Banach space, the Grothendieck property of $X^{*}$ is a formal consequence of the fact that each 0 -summing operator from $X$ in Hilbert space is nuclear, thus the equality $\Pi_{0}\left(X, l^{2}\right)=N\left(X, l^{2}\right)$. We give a direct proof of this fact, without using the theory of operator ideals. Denote by $v_{1}$ the nuclear norm.

Proposition 2.14. If $X$ satisfies $\Pi_{0}\left(X, l^{2}\right)=N\left(X, l^{2}\right)$, then $X^{*}$ verifies Grothendieck's theorem.

Proof. Let $T \in B\left(X^{*}, l_{n}^{2}\right)$ be induced by the sequence $\left(x_{i}\right)_{1 \leqslant i \leqslant n}$ in $X$. Take elements $\left(x_{j}^{*}\right)_{1 \leqslant j \leqslant N}$ in $X^{*}$ satisfying $\sup _{\varepsilon_{j}= \pm 1}\left\|\sum \varepsilon_{j} x_{j}^{*}\right\| \leqslant 1$. Consider a matrix $\left(a_{i j}\right)_{1 \leqslant i \leqslant n, 1 \leqslant j \leqslant N}$ such that $\sup _{j} \Sigma_{i}\left|a_{i j}\right|^{2} \leqslant 1$ and denote $M: l_{N}^{1} \rightarrow l_{n}^{2}$ the corresponding operator, for which $\|M\| \leqslant 1$. Consider the composition

$$
l_{n}^{2} \stackrel{T^{*}}{\rightarrow} X \stackrel{R}{\rightarrow} l_{N}^{1} \stackrel{M}{\rightarrow} l_{n}^{2}
$$

where $R(x)=\left(\left\langle x, x_{j}^{*}\right\rangle\right)_{1 \leqslant j \leqslant N}$. Because $B\left(l^{1}, l^{2}\right)=\Pi_{0}\left(l^{1}, l^{2}\right)$ (see introduction) the hypothesis gives

$$
\nu_{1}(M R) \leqslant C(X)\|R\| \leqslant 2 C(X)
$$

and thus

$$
\operatorname{trace}\left(M R T^{*}\right) \leqslant \nu_{1}\left(M R T^{*}\right) \leqslant 2 C(X)\|T\|
$$

But clearly

$$
\operatorname{trace}\left(M R T^{*}\right)=\sum_{i=1}^{n} \sum_{j=1}^{N} a_{i j}\left\langle x_{i}, x_{j}^{*}\right\rangle
$$

and for a suitable choice of $\left(a_{i j}\right)$, it follows

$$
\sum_{j}\left\|T\left(x_{j}^{*}\right)\right\|=\sum_{j}\left(\sum_{i}\left|\left\langle x_{i}, x_{j}^{*}\right\rangle\right|^{2}\right)^{1 / 2} \leqslant 2 C(X)\|T\| .
$$

Proposition 2.14 has no converse as will be indicated at the end of this section. The following theorem, which was conjectured in [32] (cf. Theorem 1), provides a different proof that $A^{*}$ has Grothendieck property. 
THEOREM 2.15. A 0-summing operator from the disc algebra into an arbitrary Banach space is nuclear.

Besides the lemma's presented in section 1, the proof of Theorem 2.15 requires some further observations.

LEMma 2.16. Assume $T \in \Pi_{0}\left(H^{\infty}, \eta\right)$ and $\left(\tau_{i}\right)$ a sequence of $H^{\infty}$-functions such that

$$
\left\|\sum\left|\tau_{i}\right|\right\|_{\infty}<\infty .
$$

For each $i$, define the operator $T_{i}$ by $T_{i}(\varphi)=T\left(\tau_{i} \varphi\right)$.

Then

(i) $\Sigma \pi_{p}\left(T_{i}\right) \leqslant\left\|\Sigma\left|\tau_{i}\right|\right\|_{\infty} \pi_{p}(T) \quad(0<p \leqslant 1)$

(ii) The serie $\Sigma T_{i}$ converges in $\Pi_{p}\left(H^{\infty}, Y\right) \quad(p>0)$.

Proof. It is clear that (ii) follows from (i), replacing the $\tau_{i}$ by sums of the $\tau_{i}$ on consecutive blocs.

In order to verify (i), take for each $i$ a system $\left(\varphi_{i, k}\right)$ in $H^{\infty}$ such that

$$
\sum_{k}\left|\left\langle\varphi_{i, k}, x^{*}\right\rangle\right|^{p} \leqslant 1 \text { for } x^{*} \in\left(H^{\infty}\right)^{*},\left\|x^{*}\right\| \leqslant 1
$$

and

$$
\sum_{k}\left\|T_{i}\left(\varphi_{i, k}\right)\right\|^{p} \sim \pi_{p}\left(T_{i}\right)^{p}
$$

Then, for some sequence $\left(\varrho_{i}\right)$ of positive numbers such that $\left\|\left(\varrho_{i}\right)\right\|_{l^{t / 1-p)}} \leqslant 1$ and for some $x^{*} \in\left(H^{\infty}\right)^{*},\left\|x^{*}\right\| \leqslant 1$.

$$
\begin{aligned}
\sum \pi_{p}\left(T_{i}\right) & \sim\left\{\sum_{i} \varrho_{i} \sum_{k}\left\|T_{i}\left(\varphi_{i, k}\right)\right\|^{p}\right\}^{1 / p} \\
& \leqslant \pi_{p}(T)\left\{\sum_{i, k}\left|\left\langle\varrho_{i}^{1 / p} \tau_{i} \varphi_{i, k}, x^{*}\right\rangle\right|^{p}\right\}^{1 / p} \\
& =\pi_{p}(T)\left\{\sum_{i} \varrho_{i}\left\|\tau_{i} x^{*}\right\|^{p}\left(\sum_{k}\left|\left\langle\varphi_{i, k}, \frac{\tau_{i} x^{*}}{\left\|\tau_{i} x^{*}\right\|}\right\rangle\right|^{p}\right)\right\}^{1 / p}
\end{aligned}
$$




$$
\begin{aligned}
& \leqslant \pi_{p}(T)\left(\sum_{i} \varrho_{i}\left\|\tau_{i} x^{*}\right\|^{p}\right)^{1 / p} \\
& \leqslant \pi_{p}(T) \sum\left\|\tau_{i} x^{*}\right\| \\
& \leqslant \pi_{p}(T)\left\|\sum\left|\tau_{i}\right|\right\|_{\infty}
\end{aligned}
$$

If $g$ is a function on $\Pi$ and $\theta \in \Pi$, let $g_{\theta}$ be the translate, i.e. $g_{\theta}(\psi)=g(\theta+\psi)$.

Next lemma goes back to [1] and was in slightly different form also used in [35] and [32]. The author is grateful to S. Kisliakov for an alternative proof.

Lemma 2.17. Assume $T \in \Pi_{0}(A, Y)$. Then:

(i) The functions $\xi_{r}(\theta)=T\left(\mathscr{R}\left(P_{r}, \theta\right)\right)$ converge in $L_{r}^{p}(\Pi)$ for $r \rightarrow 1$, for $p<1$.

(ii) If $F(\theta)=\sup _{r<1}\left\|\xi_{r}(\theta)\right\|$, then $F$ verifies the weak type inequality

$$
\|F\|_{W} \leqslant C(p) \pi_{p}(T) \quad(0<p<1) .
$$

Proof. Fix $0<p<1$. The Pietch theorem yields a Radon probability measure $\Omega$ on the closed unit ball of $C(\Pi)^{*}$ such that for $\varphi \in A$

$$
\|T(\varphi)\| \leqslant \pi_{p}(T)\left\{\int\left|x^{*}(\varphi)\right|^{p} \Omega\left(d x^{*}\right)\right\}^{1 / p} .
$$

Given $x^{*} \in C(\Pi)^{*}$, denote $x_{1}^{*}$ the image measure of $x^{*}$ for the map $t \mapsto(-t)$. Observe that $x^{*}\left(\mathscr{R}\left(P_{r, \theta}\right)\right)=\left[\mathscr{R}\left(x_{1}^{* *} P_{r}\right)\right](\theta)$. For $\theta \in \Pi$ and $0 \leqslant r, s<1$, it follows

$$
\left\|\xi_{r}(\theta)-\xi_{s}(\theta)\right\|^{p} \leqslant \pi_{p}(T)^{p} \int\left|\mathscr{R}\left(\left(x_{1}^{*} * P_{r}\right)-\left(x_{1}^{*} * P_{s}\right)\right)(\theta)\right|^{p} \Omega\left(d x^{*}\right)
$$

and

$$
\left\|\xi_{r}-\xi_{s}\right\|_{L_{Y}^{p}} \leqslant \pi_{p}(T)\left\{\int\left\|\mathscr{R}\left(\left(x_{1}^{*} * P_{r}\right)-\left(x_{1}^{*} * P_{s}\right)\right)\right\|_{p}^{p} \Omega\left(d x^{*}\right)\right\}^{1 / p}
$$

Thus (i) follows from Proposition 1:1 and the Lebesgue dominated convergence theorem. Further

$$
\sup _{r<1}\left\|\xi_{r}(\theta)\right\|^{p} \leqslant \pi_{p}(T)^{p} \int \sup _{r}\left|\mathscr{R}\left(x_{1}^{*} * P_{r}\right)(\theta)\right|^{p} \Omega\left(d x^{*}\right)
$$


and hence, taking

$$
\int F^{p} \omega \leqslant \pi_{p}(T)^{p} \iint\left(\sup _{r}\left|\mathscr{R}\left(x_{1}^{*} * \chi_{r}\right)(\theta)\right|\right)^{p} \omega(\theta) \Omega\left(d x^{*}\right) m(d \theta) .
$$

By Proposition 1.1 and Lemma 1.2, we can estimate for fixed $x^{*}$

$$
\begin{aligned}
\int\left(\sup _{r}\left|\mathscr{R}\left(x_{1}^{* *} P_{r}\right)(\theta)\right|\right)^{p} \omega(\theta) d \theta & \leqslant \frac{2}{1-p}\|\omega\|_{1}^{1-p}\left\|\sup _{r}\left|\mathscr{R}\left(x_{1}^{* *} P_{r}\right)\right|\right\|_{W}^{p} \\
& \leqslant \frac{C}{1-p}\|\omega\|_{1}^{1-p} .
\end{aligned}
$$

Hence

$$
\lambda^{p}\|\omega\|_{1} \leqslant \pi_{p}(T)^{p} \frac{C}{1-p}\|\omega\|_{1}^{1-p}
$$

and finally

$$
\lambda\|\omega\|_{1} \leqslant\left(\frac{C}{1-p}\right)^{1 / p} \pi_{p}(T) .
$$

completing the proof.

To prove Theorem 2.15, we proceed again by decomposition. Fix $0<p<1$. We show that for each $\delta>0$ there is a constant $K_{\delta}<\infty$ such that any $T \in \Pi_{p}(A, Y)$ decomposes as $T=N+R$, where

(i) $N \in N(A, Y)$ and $v_{1}(N) \leqslant K_{\delta} \pi_{p}(T)$

(ii) $\pi_{p}(R)<\delta \cdot \pi_{p}(T)$.

Fixing $0<\delta<1$ and iterating, an estimation $\nu_{1}(T) \leqslant C \pi_{p}(T)$ then follows.

By the second remark after Proposition 2.1, we can assume that $\pi_{1}(T)$ is realized by an $m$-regular Pietch measure on $\Pi$ and hence is defined on $H^{\infty}$.

Consider again a Radon probability measure $\Omega$ on the unit ball of $C(\Pi)^{*}$ for which

$$
\|T(\varphi)\| \leqslant \pi_{p}(T)\left\{\int\left|x^{*}(\varphi)\right|^{p} \Omega\left(d x^{*}\right)\right\}^{1 / p} \text { for } \varphi \in A .
$$

Denoting $\left|x^{*}\right|$ the variation of the measure $x^{*}$, consider following measure on $\Pi$

$$
\mu=\int\left|x^{*}\right| \Omega\left(d x^{*}\right)
$$

Thus $\mu$ is positive and $\|\mu\| \leqslant 1$. Let $d \mu=f d m+d \mu_{s}$ be the Lebesgue decomposition. 
Fixing $\delta>0$, apply Proposition 1.7 to $f$, giving the sequences $\left(t_{i}\right),\left(\theta_{i}\right)$ in $H^{\infty}$ and $\left(c_{i}\right) \geqslant 0$.

If for each $i$, we define the operator $T_{i}(\varphi)=T\left(\theta_{i} \tau_{i} \varphi\right)$, Lemma 2.16 implies

$$
\sum \pi_{p}\left(T_{i}\right) \leqslant C \pi_{p}(T)
$$

If $F_{i}(\theta)=\sup _{r<1}\left\|T_{i}\left(\mathscr{R}\left(P_{r,-\theta}\right)\right)\right\|$, application of Lemma 2.17 gives

$$
\left\|F_{i}\right\|_{W} \leqslant C(p) \pi_{p}\left(T_{i}\right)
$$

Take $\lambda_{i}=c_{i} \pi_{p}(T) / \delta$ and apply Lemma 1.5 to $F_{i}$ to obtain an $H^{\infty}$-function $K_{i}$ such that

$$
\begin{aligned}
\left\|x_{i}\right\|_{\infty} & \leqslant 3 \\
F_{i}\left|x_{i}\right| & \leqslant \lambda_{i} \\
\left\|1-x_{i}\right\|_{1} & \leqslant C \lambda_{i}^{-1}\left\|F_{i}\right\|_{w} .
\end{aligned}
$$

Define now

$$
\begin{gathered}
N_{i}(\varphi)=T_{i}\left(\tau_{i} x_{i} \varphi\right) \\
N=\sum_{i} N_{i} \\
R=T-N .
\end{gathered}
$$

For $0 \leqslant r<1$, consider the operator $S_{i, r}(\varphi)=T_{i}\left(\mathscr{R}\left(\tau_{i} x_{i} \varphi\right) * P_{r}\right)$ on $C(\Pi)$.

Since

$$
\left(\tau_{i} \varkappa_{i} \varphi\right) * P_{r}=\int\left(\tau_{i} \varkappa_{i} \varphi\right)(\psi) P_{r,-\psi} m(d \psi)
$$

we get

$$
\left\|S_{i, r}(\varphi)\right\| \leqslant \int\left|\left(\tau_{i} \varkappa_{i} \varphi\right)(\psi)\right|\left\|T_{i}\left(\mathscr{R}\left(P_{r,-\psi}\right)\right)\right\| \leqslant \int|\varphi(\psi)|\left(\left|\tau_{i} \varkappa_{i}\right| F_{i}\right)(\psi)
$$

Hence

$$
\pi_{1}\left(S_{i, r}\right) \leqslant\left\|\tau_{i} \varkappa_{i} F_{i}\right\|_{1} \leqslant \lambda_{i}\left\|\tau_{i}\right\|_{1}=\frac{\pi_{p}(T)}{\delta} c_{i}\left\|\tau_{i}\right\|_{1} .
$$


Also, for $0 \leqslant r, s<1$

$$
\begin{aligned}
\pi_{1}\left(S_{i, r}-S_{i, s}\right) & \leqslant \int\left|\left(\tau_{i} \varkappa_{i}\right)(\psi)\right|\left\|T_{i}\left(\mathscr{R}\left(P_{r,-\psi}\right)\right)-T_{i}\left(\mathscr{R}\left(P_{s,-\psi}\right)\right)\right\| \\
& \leqslant C \int\left|\varkappa_{i}(\psi)\right| F_{i}(\psi)^{1-p}\left\|T_{i}\left(\mathscr{R}\left(P_{r,-\psi}\right)\right)-T_{i}\left(\mathscr{R}\left(P_{s,-\psi}\right)\right)\right\|^{p} \\
& \leqslant C \lambda_{i}^{1-p} \int\left\|T_{i}\left(\mathscr{R}\left(P_{r, \psi}\right)\right)-T_{i}\left(\mathscr{R}\left(P_{s, \psi}\right)\right)\right\|^{p}
\end{aligned}
$$

and Lemma 2.17 implies the convergence of $\left(S_{i, r}\right)$ in $\Pi_{1}(C(I), Y)$ for $r \rightarrow 1$. Since the $S_{i, r}$ are clearly nuclear, the limit operator $S_{i}$ will also be nuclear and

$$
v_{1}\left(S_{i}\right)=\pi_{1}\left(S_{i}\right) \leqslant \frac{\pi_{p}(T)}{\delta} c_{i}\left\|\tau_{i}\right\|_{1}
$$

Since $S_{i}$ extends $N_{i}$, we obtain $N \in N(A, Y)$ and

$$
\nu_{1}(N) \leqslant \sum_{i} v_{1}\left(N_{i}\right) \leqslant \frac{\pi_{p}^{\cdot}(T)}{\delta} \sum c_{i}\left\|\tau_{i}\right\|_{1} \leqslant C \delta^{-C-1} \pi_{p}(T) .
$$

So it remains to estimate $\pi_{p}(R)$. Let for convenience $\eta=1-\sum \theta_{i} \tau_{i}^{2} x_{i}$.

Take a sequence $\left(\psi_{k}\right)$ in $A$ such that

(i) $\left\|\psi_{k}\right\|_{\infty} \leqslant 1$

(ii) $\int\left|\psi_{k}\right| d \mu_{s} \rightarrow 0$

(iii) $\psi_{k} \rightarrow 1 \quad m-$ a.e.

Consider for each $k$ the operator $R_{k}(\varphi)=R\left(\varphi \psi_{k}\right)$. Then

$$
\left\|R_{k}(\varphi)\right\|=\left\|T\left(\left(1-\sum \theta_{i} \tau_{i}^{2} \varkappa_{i}\right) \varphi \psi_{k}\right)\right\|=\lim _{r \mathbb{l}^{1}}\left\|T\left(\left(\eta * P_{r}\right) \varphi \psi_{k}\right)\right\|
$$

and

$$
\begin{aligned}
\left\|T\left(\left(\eta * P_{r}\right) \varphi \psi_{k}\right)\right\| & \leqslant \pi_{p}(T)\left\{\int\left|\left\langle\left(\eta * P_{r}\right) \varphi \psi_{k}, x^{*}\right\rangle\right|^{p} \Omega\left(d x^{*}\right)\right\}^{1 / p} \\
& =\pi_{p}(T)\left\{\int\left|\left\langle\varphi, \frac{\left(\eta * P_{r}\right) \psi_{k} x^{*}}{\left\|\left(\eta * P_{r}\right) \psi_{k} x^{*}\right\|}\right\rangle\right|^{p}\left\|\left(\eta * P_{r}\right) \psi_{k} x^{*}\right\|^{p} d \Omega\right\}^{1 / p}
\end{aligned}
$$


Hence

$$
\pi_{p}\left(R_{k}\right) \leqslant \pi_{p}(T) \varliminf_{r \varliminf^{1}}\left\{\int\left\|\left(\eta * P_{r}\right) \psi_{k} x^{*}\right\|^{p} \Omega\left(d x^{*}\right)\right\}^{1 / p}
$$

where the second factor is dominated by

$$
\varliminf_{r \gtrless^{1}} \int\left(|\eta| * P_{r}\right)\left|\psi_{k}\right| d \mu
$$

Since $\lim _{k \rightarrow \infty}\left\|R-R_{k}\right\|=0$, we get

$$
\pi_{p}(R) \leqslant \frac{\varliminf_{k}}{k} \pi_{p}\left(R_{k}\right) \leqslant\left\{\int\left|1-\sum \theta_{i} \tau_{i}^{2} x_{i}\right| f d m\right\} \pi_{p}(T) .
$$

Taking previous estimates in account, we see that the latter quantity is bounded by

$$
\begin{gathered}
\int\left|1-\sum \theta_{i} \tau_{i}^{2}\right| f+C \sum \int\left|1-x_{i}\right|\left|\tau_{i}\right| f \\
\leqslant \delta+C \sum c_{i}\left\|1-x_{i}\right\|_{1} \\
\leqslant \delta+C \frac{\delta}{\pi_{p}(T)} \sum\left\|F_{i}\right\|_{w} \\
\leqslant \delta+C(p) \delta .
\end{gathered}
$$

This establishes Theorem 2.15 .

Theorem 2.15 permits to distinguish the disc algebra from certain other translation invariant spaces. Recall that a subset $\Lambda$ of $Z$ is a $\Lambda_{p}$-set provided $L^{1}$ and $L^{p}$-norms are equivalent on linear combinations of the characters $e^{i n t}$ with $n \in \Lambda$. Combining Theorem 2.15 with Lemma 1 of [30], following result is derived.

COROLlaRY 2.18. Assume $\Lambda \subset Z$ such that $\Lambda \cap Z_{-}$is a $\Lambda_{p}$-set for some $p>1$. Then the space $C_{\Lambda}$, closure in $C(I)$ of the polynomials $f=\Sigma_{n} \in \Lambda c_{n} e^{i n t}$, is not a quotient of the disc algebra.

\subsection{Remarks}

(1) In proving the interpolation inequality in Proposition 2.3 , the norms $\pi_{p}(T)$ and $\pi_{q}(T)$ were computed using different measures. It may be possible to derive the result from weighted norm inequalities on the Hilbert transform, using less the algebra structure. 
(2) It is shown in [13] that the spaces $L_{\{0,1, \ldots, n\}}^{\infty}$ of polynomials of degree $\leqslant n$ embed uniformly complementedly in $A$. Hence, previous results localize to these polynomial spaces. It is also proved in [13] that the Banach-Mazur distance $d\left(l_{n+1}^{\infty}, L_{\{0,1, \ldots, n\}}^{\infty}\right) \leqslant C \log n$, while for an arbitrary finite subset $\Lambda$ of $\mathbf{Z}$, one always has that $L_{\Lambda}^{\infty}$ is only a $P_{\lambda}$-space for $\lambda$ of order $\log |\Lambda|$. So Corollary 2.6 is sharp. Other examples of complemented subspaces of $A$ are those obtained by spline interpolation in [3].

(3) Assume $\Lambda \subset Z$ such that $Z_{+} \subset \Lambda$ and $\Lambda \cap Z_{-}$is a Hadamard lacunary set. From the result on the disc algebra, it is then straight forward to show that also $B\left(C_{\Lambda}, l^{1}\right)=$ $\Pi_{2}\left(C_{\Lambda}, l^{1}\right)$. On the other hand, as we explained in the introduction, the orthogonal projection from $C_{\Lambda}$ on $L_{\Lambda \cap z_{-}}^{2}$ is 0 -summing and onto. Consequently, the previous property does not imply nuclearity, even in case of translation invariant spaces. The reader will find related results in [32], section 3.

(4) It should be mostly interesting to determine for what spaces $X$ it is true that any operator from $A$ into $X$ can be extended to $C(\Pi)$. This property is obviously true for $X=l^{\infty}$ and, by our results, if $X$ has a finite cotype (the extreme case in the other sense). The case $X=B\left(l^{2}, l^{2}\right)$ is unsettled and a positive solution will have applications in operator theory.

\section{An interpolation result for vector valued $\boldsymbol{H}^{\mathbf{1}}$-spaces}

The purpose of this section is to characterize certain spaces $H_{X}^{1}$. Our motivation for studying such spaces was to simplify earlier work on the minimum-norm lifting $L^{1} / H_{0}^{1} \leadsto L^{1}$ by using the interpolating sequence theory. The results presented in the first paragraph can be extended in the frame of the Lions-Peetre interpolation theory. There are also possibly other applications than those considered here.

\subsection{Characterization of certain vector-valued $H^{1}$ functions}

Our purpose is to prove Theorem 0.3. In what follows, Proposition 1.7 will be again important.

We first show the following extension of Proposition 1.6.

LEMMA 3.1. Given $f \in L_{+}^{1}(\Pi)$ and $\delta>0$, there exists $\tilde{f} \in L_{+}^{1}(\Pi)$ and $\varphi \in H^{\infty}$ satisfying the following conditions:

(i) $\|\varphi\|_{\infty} \leqslant C$

(ii) $\left\|\tilde{f}_{1} \leqslant C \delta^{-C}\right\| f \|_{1}$ 
(iii) $\int_{\Pi}|1-\varphi| f d m \leqslant \delta\|f\|_{1}$

(iv) given $F \in H^{1}$, there is a decomposition $F \varphi=F_{1}+F_{2}$ in $H^{1}$, where

$$
\left|F_{1}\right| \leqslant f \text { and }\left\|F_{2}\right\|_{1} \leqslant C \int_{\||F| \geqslant f]}|F| \text {. }
$$

Proof. Apply first Lemma 1.7 and put $\varphi=\Sigma \theta_{i} \tau_{i}^{2}$ and $\tilde{f}=\Sigma c_{i}\left|\tau_{i}\right|$. Then (i), (ii), (iii) hold.

Next, apply for fixed $i \in Z$ Lemma 1.6 to the $H^{1}$ function $\tau_{i} F$, taking $\lambda=c_{i}$. This gives a decomposition in $H^{1}$

$$
\tau_{i} F=F_{i}^{1}+F_{i}^{2}
$$

where

$$
\left|F_{i}^{1}\right| \leqslant C c_{1} \text { and }\left\|F_{i}^{2}\right\|_{1} \leqslant C \int_{\left[\left|z_{i} F\right| \geq c_{i}\right]}\left|\tau_{i} F\right|
$$

Notice that by (iii) of Proposition 1.7

$$
\int_{\left[\left|\tau_{F}\right| \geqslant c_{i}\right]}\left|\tau_{i} F\right| \leqslant \int_{[|F| \geq f]}^{\cdot}\left|\tau_{i}\right||F| .
$$

If we let

$$
F_{1}=\sum \theta_{i} \tau_{i} F_{i}^{1} \text { and } F_{2}=\sum \theta_{i} \tau_{i} F_{i}^{2}
$$

then obviously

$$
\left|F_{1}\right| \leqslant C \sum c_{i}\left|\tau_{i}\right|
$$

and

$$
\left\|F_{2}\right\|_{1} \leqslant \sum C\left\|F_{i}^{2}\right\|_{1} \leqslant C \int_{[|F| \geqslant f]}\left(\sum\left|\tau_{i}\right|\right)|F|
$$

Thus the decomposition $F \varphi=F_{1}+F_{2}$ satisfies (iv).

Let us now fix some terminology. 
If $X_{0}, X_{1}$ are linear subspaces of a vector space $X$ and \|\|$_{0},\|\|_{1}$ norms on $X_{0}, X_{1}$ respectively, we equip

$$
X_{0} \cap X_{1} \text { with the norm }\|x\|_{X_{0} \cap X_{1}}=\max \left(\|x\|_{0},\|x\|_{1}\right)
$$

and

$$
X_{0} \cup X_{1}=\left\{x \in X, x=x_{0}+x_{1} \text { for some } x_{0} \in X_{0}, x_{1} \in X_{1}\right\}
$$

with the norm

$$
\|x\|_{X_{0} \cup X_{1}}=\inf _{x=x_{0}+x_{1}}\left(\left\|x_{0}\right\|_{0}+\left\|x_{1}\right\|_{1}\right)
$$

Fix a positive integer $N$ and $\varepsilon>0$.

Let $X_{0}$ be $C^{N}$ equipped with sup-norm and $X_{1}$ obtained by defining on $\mathrm{C}^{N}$ the norm

$$
\left\|\left(z_{1}, \ldots, z_{N}\right)\right\|_{1}=\varepsilon \sum_{k}\left|z_{k}\right|
$$

We will use the following simple fact:

LEMMA 3.2. For given $x=\left(z_{1}, \ldots, z_{N}\right) \in \mathbf{C}^{N}$, define $x^{\prime}=\left(z_{1}^{\prime}, \ldots, z_{N}^{\prime}\right)$ by

$$
\left\{\begin{array}{l}
z_{k}^{\prime}=z_{k} \text { if }\left|z_{k}\right| \geqslant 2\|x\|_{X_{0} \cup X_{1}} \\
z_{k}^{\prime}=0 \text { otherwise. }
\end{array}\right.
$$

Then $\left\|x^{\prime}\right\|_{1} \leqslant 2\|x\|_{X_{0} \cup X_{1}}$.

Proof. Let $x=x_{0}+x_{1}$ where $x_{0}=\left(z_{1,0}, \ldots, z_{N, 0}\right), x_{1}=\left(z_{1,1}, \ldots, z_{N, 1}\right)$ are such that $\|x\|_{X_{0} \cup X_{1}}=\left\|x_{0}\right\|_{0}+\left\|x_{1}\right\|_{1}$. If $\left|z_{k}\right| \geqslant 2\|x\|_{X_{0} \cup X_{1}}$, then clearly

$$
\left|z_{k, 1}\right| \geqslant\left|z_{k}\right|-\left|z_{k, 0}\right| \geqslant\left|z_{k}\right|-|| x_{0}\left\|_{0} \geqslant\left|z_{k}\right|-|| x\right\|_{X_{0} \cup X_{1}} \geqslant \frac{1}{2}\left|z_{k}\right|
$$

and hence

$$
\|x\|_{X_{0} \cup X_{1}} \geqslant\left\|x_{1}\right\|_{1} \geqslant \frac{\varepsilon}{2} \sum\left|z_{k}^{\prime}\right|
$$

proving the lemma.

If $X$ is a Banach space, denote for $1 \leqslant p \leqslant \infty$ by $H_{X}^{p}$ the subspace of $L_{X}^{p}$ of functions $f$ such that $f(n)=0$ if $n<0$. 
Let us prove Theorem 0.3 .

PROPOSITION 3.3. The norms of the spaces $H_{X_{0}}^{1} \cup H_{X_{1}}^{1}$ and $H_{X_{0} \cup X_{1}}^{1}$ are equivalent up to a fixed constant (which does not depend on $N$ or $\varepsilon$ ).

Proof. The $H_{X_{0}}^{1} \cup H_{X_{1}}^{1}$ norm clearly dominates the $H_{X_{0} \cup X_{1}}^{1}$ norm, since if $\xi_{0}+\xi_{1}$, then

$$
\begin{aligned}
\int\left\|\xi\left(e^{i \theta}\right)\right\|_{X_{0} \cup X_{1}} & \leqslant \int\left\|\xi_{0}\left(e^{i \theta}\right)\right\|_{X_{0}}+\int\left\|\xi_{1}\left(e^{i \theta}\right)\right\|_{X_{1}} \\
& =\left\|\xi_{0}\right\|_{H_{X_{0}}^{1}}+\left\|\xi_{1}\right\|_{H_{X_{1}}^{1}} .
\end{aligned}
$$

Conversely, assume $\xi=\left(F_{1}, \ldots, F_{N}\right)$ in $H_{X_{0} \cup X_{1}}^{1}$ and define $f$ by

$$
f(\vartheta)=2\left\|\left(F_{1}(\vartheta), \ldots, F_{N}(\vartheta)\right)\right\|_{X_{0} \cup X_{1}} \text { for } \vartheta \in \Pi .
$$

Fixing $\delta>0$, take $\tilde{f}$ and $\varphi$ as in Lemma 3.1. For $k=1, \ldots, N$, let further

$$
F_{k} \varphi=F_{k, 0}+F_{k, 1}
$$

be an $H^{1}$-decomposition satisfying

$$
\left|F_{k, 0}\right| \leqslant \bar{f} \text { and }\left\|F_{k, 1}\right\|_{1} \leqslant C \int_{\left[F_{k} \mid \geqslant f\right]}\left|F_{k}\right| .
$$

Define $\xi_{0}=\left(F_{1,0}, \ldots, F_{N, 0}\right)$ and $\xi_{1}=\left(F_{1,1}, \ldots, F_{N, 1}\right)$. Then by Lemma 3.1 (ii)

$$
\left\|\xi_{0}\right\|_{H_{X_{0}}^{1}}=\int \max _{k}\left|F_{k, 0}\right| \leqslant\|\tilde{f}\|_{1} \leqslant 2 C \delta^{-C}\|\xi\|_{H_{X_{0} \cup X_{1}}^{1}}
$$

and using Lemma 3.2

$$
\begin{aligned}
\left\|\xi_{1}\right\|_{H_{X_{1}}^{1}}=\varepsilon \sum_{k}\left\|F_{k, 1}\right\|_{1} & \leqslant C \int \varepsilon \sum_{k}\left|F_{k}\right| \chi_{\left[F_{k} \mid \geqslant f\right]} \\
& \leqslant 2 C \int\left\|\left(F_{1}, \ldots, F_{N}\right)\right\|_{X_{0} \cup X_{1}} .
\end{aligned}
$$

Finally, by Lemma 3.1 (iii)

$$
\begin{aligned}
\left\|\xi-\left(\xi_{0}+\xi_{1}\right)\right\|_{H_{X_{0} \cup X_{1}}^{1}} & =\int|1-\varphi|\left\|\left(F_{1}, \ldots, F_{N}\right)\right\|_{X_{0} \cup X_{1}}=\frac{1}{2} \int|1-\varphi| f \\
& \leqslant \delta\|\xi\|_{H_{X_{0} \cup X_{1}}^{1}} .
\end{aligned}
$$

3-848288 Acta Mathematica 152. Imprimé le 17 Avril 1984 
For $\varrho>0$, denote $\mathscr{B}_{\varrho}$ the ball with midpoint 0 and radius $\varrho$ in $H_{X_{0} \cup X_{1}}^{1}$. From the preceding, it follows that $\mathscr{B}_{1}$ is contained in

$$
\left\{\zeta \in H_{X_{0}}^{1} \cup H_{X_{1}}^{1}:\|\xi\|_{H_{X_{0}}^{1} \cup H_{X_{1}}^{1}} \leqslant 2 C \delta^{-C}+2 C\right\}+\beta_{\delta} .
$$

Choosing $\delta<1$, we conclude that

$$
\|\zeta\|_{H_{X_{0}}^{1} \cup H_{X_{1}}^{1}} \leqslant \text { const. }\|\xi\|_{H_{X_{0} \cup X_{1}}^{1}} .
$$

Dualization of Proposition 3.3 (in which one can obviously replace $H^{1}$ by $H_{0}^{1}$ ) leads to

PROPOSITION 3.4. The norms of the spaces $L_{X_{0}^{*} / \mathscr{H}}^{\infty} \cap L_{X_{1}^{*} / \mathscr{H}}^{\infty}$ and $L_{X_{0}^{*} \cap X_{1}^{*} / \mathscr{H}}^{\infty}$ are equivalent up to a fixed constant.

We denote here by $\mathscr{H}$ the subspace of $L_{\mathbf{C}^{N}}^{\infty}$ of those elements which have $H^{\infty}$ components.

\subsection{Application to interpolating sequences in the disc}

Proposition 3.4 can be applied to obtain certain P. Beurling type functions.

Consider the following vector-valued interpolation problem. Let $N$ be a fixed positive integer and $\mathscr{E}_{1}, \mathscr{E}_{2}, \ldots, \mathscr{E}_{N}$ (finite) subsets of the open disc $D$. Let further for each $k=1, \ldots, N$ a complex valued function $\gamma_{k}$ on $\mathscr{E}_{k}$ be given. Consider

$$
\Phi=\left(\varphi_{1}, \ldots, \varphi_{N}\right) \text { such that } \varphi_{k} \in H^{\infty} \text { and } \varphi_{k} \mid \mathscr{E}_{k}=\gamma_{k}
$$

Let now $X$ be $\mathbf{C}^{N}$ equipped with an unconditional norm. Define

$$
\sigma_{X}=\inf \|\Phi\|_{H_{X}^{\infty}}
$$

where the infimum is taken over all $\Phi$ satisfying $\left({ }^{*}\right)$.

Let $B_{k}$ be the Blashke-product of the points in $\mathscr{E}_{k}$. If $\Phi$ is a particular solution of $\left({ }^{*}\right)$, the general solution becomes

$$
\Psi=\left(\psi_{1}, \ldots, \psi_{N}\right) \text { where } \psi_{k}=\varphi_{k}+B_{k} \cdot \omega_{k} \text { and } \omega_{k} \in H^{\infty} .
$$

By unconditionality of $X$, this fact leads to the formula

$$
\sigma_{X}=\left\|\left(\frac{\varphi_{1}}{B_{1}}, \ldots, \frac{\varphi_{N}}{B_{N}}\right)\right\|_{L_{X}^{\infty} / \mathscr{H}}
$$

If $X_{0}, X_{1}$ are as above, Proposition 1.4 leads to the following result: 
Proposition 3.5. $\sigma_{X_{0}^{*} \cap X_{1}^{*}} \leqslant K \max \left(\sigma_{X_{0}^{*}}, \sigma_{X_{1}^{*}}\right)$, for some numerical $K$.

This property can be restated as follows.

COROLLARY 3.6. If $\Phi_{0}, \Phi_{1}$ are solutions of $\left({ }^{*}\right)$, then $\left({ }^{*}\right)$ has a solution $\Phi$ for which

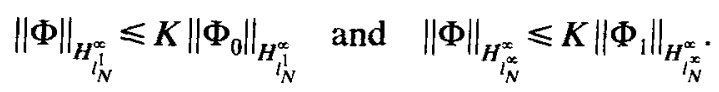

Thus in solving $\left({ }^{*}\right)$, information on $l^{\infty}$ - and $l^{1}$-estimations can always be combined.

In particular, one has

COROLlaRY 3.7. Assume $\mathscr{E}_{1}, \ldots, \mathscr{E}_{N}$ subsets of $D$ for which there exist $H^{\infty}$. functions $\varphi_{1}, \ldots, \varphi_{N}$ satisfying

(i) $\varphi_{k}(z)=1$ for each $z \in \mathscr{E}_{k}$ and $k=1, \ldots, n$

(ii) $\left\|\Sigma\left|\varphi_{k}\right|\right\|_{\infty} \leqslant M$.

Then there exist also $\boldsymbol{H}^{\infty}$-functions $\psi_{1}, \ldots, \psi_{N}$ fulfilling (i) and moreover

(iii) $\left\|\psi_{k}\right\|_{\infty} \leqslant K$ for each $k=1, \ldots, n$

(iv) $\left\|\Sigma\left|\psi_{k}\right|\right\|_{\infty} \leqslant K M$.

Recall that a sequence $\left(z_{n}\right)$ in the open unit disc is $\delta$-interpolating $(\delta>0)$ provided to each $\delta$-bounded sequence $\left(a_{n}\right)$ of complex numbers corresponds some $\varphi \in H^{\infty}$ with $\|\varphi\|_{\infty} \leqslant 1$ and $\varphi\left(z_{n}\right)=a_{n}$ for each $n$. $A$ result of $L$. Carleson asserts that a sequence $\left(z_{n}\right)$ is interpolating if and only if the sequence is uniformly separated, i.e.

$$
\inf _{m} \prod_{n \neq m} d\left(z_{n}, z_{m}\right)>0 \quad \text { where } \quad d(z, w)=\left|\frac{z-w}{1-\bar{z} w}\right|
$$

(see [14]). For $z \in D$, denote $\delta_{z}$ its Dirac measure. The sequence $\left(z_{n}\right)$ is called a Carleson sequence provided the measure $\mu=\Sigma\left(1-\left|z_{n}\right|\right) \delta_{z_{n}}$ is a Carleson measure on $D$ (see [20], p. 31 for definition). The constant of the Carleson sequence $\left(z_{n}\right)$ is the Carleson norm of $\mu$.

A sequence in $D$ is known to be Carleson iff it is finite union of interpolating sequences. If $\left(z_{n}\right)$ is interpolating, then, by a result of $P$. Beurling, there is a sequence $\left(\varphi_{n}\right)$ in $H^{\infty}$ satisfying

(i) $\left\|\Sigma\left|\varphi_{n}\right|\right\|_{\infty}<\infty$

(ii) $\varphi_{m}\left(z_{n}\right)=\delta_{m, n}$ (Kronecker's symbol). 
For an explicit formula for the functions $\varphi_{n}$, see [28] (Theorem 1). In the next section, we will make use of the following consequence of Corollary 3.7.

COROLLARY 3.8. Given $M<\infty$ there is $M_{1}<\infty$ such that if $\mathfrak{F}_{1}, \mathfrak{F}_{2}, \ldots, \mathfrak{E}_{N}$ is a partition of a Carleson sequence of constant $M$, then there exist $H^{\infty}$-functions $\varphi_{1}, \varphi_{2}, \ldots, \varphi_{N}$ satisfying

(i) $\varphi_{k}(z)=1$ for each $z \in \mathfrak{F}_{k}$ and $k=1,2, \ldots, N$

(ii) $\left\|\varphi_{k}\right\|_{\infty} \leqslant K$ for each $k=1,2, \ldots, N$

(iii) $\left\|\Sigma\left|\varphi_{k}\right|\right\|_{\infty} \leqslant M_{1}$.

The important thing is that $K$ does not depend on $M$. A slightly weaker version of previous result was obtained in [8] by different techniques.

\section{Properties of the minimum norm lifting}

Let us repeat that the minimum-norm lifting $\sigma: L^{1} / H_{0}^{1} \leadsto L^{1}$ maps $x \in L^{1 /} / H_{0}^{1}$ on the unique $f \in L^{1}$ satisfying $\|x\|=\|f\|_{1}$ and $q(f)=x$.

If $A \subset L^{1} / H_{0}^{1}$ is a WCC set (see introduction), then $\sigma(A)$ is relatively weakly compact ([44], Theorem 7.1). The purpose of this section is to prove a local version of this property. The following result implies Theorem 0.4 .

THEOREM 4.1. For each $\delta>0$ there exists $\delta_{1}>0$ such that given $L^{1}(\Pi)$-functions $f_{1}, f_{2}, \ldots, f_{n}$ satisfying the following conditions:

(i) $\left\|q\left(f_{m}\right)\right\|>\left(1-\delta^{2}\right)\left\|f_{m}\right\|_{1} \quad$ for $1 \leqslant m \leqslant n$

(ii) $\int \max _{m} \lambda_{m}\left|f_{m}\right| \geqslant C \delta \Sigma \lambda_{m}\left\|f_{m}\right\|_{1}$ whenever $\lambda_{m} \geqslant 0$,

then there are $H^{\infty}$-functions $g_{1}, g_{2}, \ldots, g_{n}$ such that

(iii) $\left|g_{1}\right|+\left|g_{2}\right|+\ldots+\left|g_{n}\right| \leqslant 1$ pointwise on $\Pi$.

(iv) $\left\langle f_{m}, g_{m}\right\rangle=\int f_{m} g_{m}=\delta_{1}\left\|f_{m}\right\|_{1}$ for $1 \leqslant m \leqslant n$.

Condition (ii) of Theorem 4.1 also means that the $f_{m}$ have mass at least $C \delta\left\|f_{m}\right\|_{1}$ on disjoint subsets of $\Pi$ (cf. [17], Proposition 2.2). We will derive Theorem 4.1 as a consequence of Corollary 3.8 . The author obtained the result previously by a more direct method.

Next lemma, based on an argument of successive extractions, is left as an exercice. 
LEMMA 4.2. Given $\varrho>0, x>0$, there exists $\eta=\eta(\varrho, x)>0$ such that if $\left(\alpha_{m}\right)_{1 \leqslant m \leqslant n}$ are positive functions in $L^{1}(\Pi)$ and

$$
\int \max _{m} \alpha_{m} \geqslant \varrho \sum_{m=1}^{n}\left\|\alpha_{m}\right\|_{1}
$$

one can find a subset $S$ of $\{1,2, \ldots, n\}$ and a system $\left(A_{m}\right)_{m \in S}$ of disjoint measurable subsets of $\Pi$ satisfying

(i) $\Sigma_{m \in S}\left\|\alpha_{m}\right\|_{1} \geqslant \eta \sum_{m=1}^{n}\left\|\alpha_{m}\right\|_{1}$

(ii) $\int_{A_{m}} \alpha_{m} \geqslant(\varrho / 2)\left\|\alpha_{m}\right\|_{1}$ for each $m \in S$

(iii) $\int \max _{m \in S}\left(\alpha_{m} \chi_{\Pi \backslash A_{m}}\right) \leqslant \varkappa \int \max _{m \in S} \alpha_{m}$.

Another elementary fact needed for the proof of Theorem 4.1 is the following approximation principle.

LEMMA 4.3. To each $\varepsilon>0$ corresponds $\gamma=\gamma(\varepsilon)>0$ such that for positive, disjointly supported $L^{1}(\Pi)$-functions $\alpha_{1}, \alpha_{2}, \ldots, \alpha_{n}$ of norm 1 , there exist functions $\alpha_{1}^{\prime}, \alpha_{2}^{\prime}, \ldots, \alpha_{n}^{\prime}$ such that

(i) $\left\|\alpha_{m}-\alpha_{m}^{\prime}\right\|_{1}<\varepsilon$ for each $m$

(ii) the functions $\alpha_{m}^{\prime}$ are obtained by taking disjoint convex combinations of the Poisson-kernels $P_{z_{k}}$, for some $\gamma$-interpolating sequence $\left(z_{k}\right)$ in $D$.

Recall that

$$
P_{z}(\theta)=\frac{1-|z|^{2}}{\left|e^{i \theta}-z\right|^{2}}
$$

Sketch of proof of Lemma 4.3. If we define for fixed $\varepsilon>0$ and positive integer $K$ for $k=0,1,2, \ldots, \varepsilon^{-1} K-1$

$$
z_{k}=\left(1-\frac{1}{K}\right) e^{i \theta_{k}} \text { where } \theta_{k}=2 \pi \varepsilon \frac{k}{K}
$$

then $\left(z_{k}\right)$ is a $\gamma$-interpolating sequence, where $\gamma$ does not depend on $K$. Also

$$
\left\|P_{z}-P_{w}\right\|_{1} \sim d(z, w) \leqslant \text { const. } \varepsilon \quad \text { if }|z|=|w|=1-\frac{1}{K} \text { and }|z-w|<\frac{\varepsilon}{K} .
$$

Choose now $K$ sufficiently large to ensure in particular that $\alpha_{m} \approx \alpha_{m} * P_{r}$ for each $m=1,2, \ldots, n$, taking $r=1-1 / K$. The functions $\alpha_{m}^{\prime}$ are then obtained by replacement of 
the Poisson-integrals by convex combination of the $P_{z_{k}}$. Since the $\alpha_{m}$ were assumed to be disjointly supported, it is clear that one can choose these combinations to be disjointly supported on the sequence $\left(z_{k}\right)$.

Proof of Theorem 4.1. We show that for some $\delta_{1}>0$ one has for $a_{1}, \ldots, a_{n} \in \mathbf{C}$

$$
\inf \int \max _{m}\left|a_{m} f_{m}+h_{m}\right| \geqslant \delta_{1} \sum\left|a_{m}\right||| f_{m} \|_{1}
$$

where the infimum is taken over all systems $\left(h_{m}\right)_{1 \leqslant m \leqslant n}$ with $h_{m} \in H_{0}^{1}$. The proof is then concluded by a Hahn-Banach extension argument. Notice that the $a_{m}$ in $\left(^{*}\right)$ can be taken positive. Let $K$ be the numerical constant appearing in Corollary 3.8 and put $\varepsilon=1 / 2 K$. Take $\gamma=\gamma(\varepsilon)$ as in Lemma 4.3 and let $M<\infty$ be such that $\gamma$-interpolating sequences are Carleson sequences of constant at most $M$. Denote $M_{1}$ be constant associated to $M$ by Corollary 3.8. Defining $\varrho=C \delta, \varkappa=\delta$, it follows from (ii) of Theorem 4.1 and Lemma 4.2 that there exist a subset $S$ of $\{1,2, \ldots, n\}$ and disjoint measurable subsets $\left(A_{m}\right)_{m \in s}$ of $\Pi$ satisfying

(i) $\Sigma_{s} \alpha_{m}\left\|f_{m}\right\|_{1} \geqslant \eta \Sigma_{m=1}^{n} a_{m}\left\|f_{m}\right\|_{1}$

(ii) $\int_{A_{m}}\left|f_{m}\right| \geqslant(C / 2) \delta|| f_{m} \|_{1}$ for each $m \in S$

(iii) $\int \max _{m \in S}\left(a_{m}\left|f_{m}\right| \chi_{\Pi \backslash A_{m}}\right) \leqslant \varkappa \int \max _{S} a_{m}\left|f_{m}\right|$.

Application of Lemma 4.3 gives a Carleson sequence $\left(z_{k}\right)$ of constant $M$ and disjoint subsets $\left(V_{m}\right)_{m \in S}$ of the index set, such that

(iv) $\left\|\left|f_{m}\right| \chi_{A_{m}}-\beta_{m}\right\|_{1}<\varepsilon\left\|f_{m} \chi_{A_{m}}\right\|_{1}(m \in S)$

where

(v) $\beta_{m} \in \omega_{m} \cdot$ convex hull $\left(P_{z_{k}} ; k \in V_{m}\right) \quad$ where $\omega_{m}=\left\|f_{m} \chi_{A_{m}}\right\|_{1}$.

Defining $\mathscr{E}_{m}=\left\{z_{k} ; k \in V_{m}\right\}$ for $m \in S$, we can choose $H^{\infty}$-functions $\left(\varphi_{m}\right)_{m \in S}$ fulfilling the conditions of Corollary 3.8.

By (i) of Theorem 4.1, there are norm-1 $H^{\infty}$-functions $\psi_{m}$ so that

(vi) $\left\langle f_{m}, \psi_{m}\right\rangle=\left(1-\delta^{2}\right)\left\|f_{m}\right\|_{1}$.

First, one deduce easily from (vi) that

$$
\int \max \left|a_{m} f_{m}+h_{m}\right| \geqslant \int \max _{S}\left|a_{m}\right| f_{m}\left|+h_{m} \psi_{m}\right|-3 \delta \sum_{S} a_{m}\left\|f_{m}\right\|_{1} .
$$


Then, by (iii) of Corollary 3.8 and (iii)

$$
\sum_{S} a_{m}\left|\int\right| f_{m}\left|\varphi_{m}\right| \geqslant \sum_{S} a_{m}\left|\int_{A_{m}}\right| f_{m}\left|\varphi_{m}\right|-\varkappa M_{1} \sum_{S} a_{m}\left\|f_{m}\right\|_{1} .
$$

Since, by (i) of Corollary 3.8

$$
\int \beta_{m} \varphi_{m}=\left\|f_{m} \chi_{A_{m}}\right\|_{1} \text { for } m \in S
$$

we deduce from (iv) and (ii) cf. Corollary 3.8

$$
\left|\int_{A_{m}}\right| f_{m}\left|\varphi_{m}\right| \geqslant(1-\varepsilon K)\left\|f_{m} \chi_{A_{m}}\right\|_{1} \quad \text { for } m \in S .
$$

Hence, combining inequalities, it follows

$$
M_{1} \int \max _{S}\left|a_{m}\right| f_{m}\left|+h_{m} \psi_{m}\right| \geqslant(1-\varepsilon K) \sum_{S} a_{m}\left\|f_{m} \chi_{A_{m}}\right\|_{1}-\chi M_{1} \sum_{S} a_{m}\left\|f_{m}\right\|_{1}
$$

and hence, using (ii) and (i)

$$
\begin{gathered}
M_{1} \int \max \left|a_{m} f_{m}+h_{m}\right| \geqslant\left(\frac{C}{4} \delta-\varkappa M_{1}-3 \delta M_{1}\right) \sum_{S} a_{m}\left\|f_{m}\right\|_{1}, \\
\int \max \left|a_{m} f_{m}+h_{m}\right| \geqslant \eta\left(\frac{C}{4 M_{1}}-4\right) \delta \sum_{m} a_{m}\left\|f_{m}\right\|_{1} .
\end{gathered}
$$

Since $M_{1}$ is a numerical constant, we can take $C=20 M_{1}$ and let $\delta_{1}=\eta \delta$.

So we obtain $\left({ }^{*}\right)$ and Theorem 4.1 is proved.

Theorem 4.1 implies clearly the following property.

COROLlARY 4.4. To each $\delta>0$ corresponds $\delta_{1}>0$ such that if $x_{1}, x_{2}, \ldots, x_{n}$ in $L^{1} / H_{0}^{1}$ satisfy

$$
\int\left\|\sum \varepsilon_{m} c_{m} \sigma\left(x_{m}\right)\right\|_{1} d \varepsilon \geqslant \delta \sum\left|c_{m}\right|\left\|x_{m}\right\|, \quad \forall\left(c_{m}\right)
$$

then also

$$
\int\left\|\sum \varepsilon_{m} c_{m} x_{m}\right\| d \varepsilon \geqslant \delta_{1} \sum\left|c_{m}\right|\left\|x_{m}\right\|, \quad \forall\left(c_{m}\right)
$$


Thus if the minimum-norm lifting of a sequence $\left(x_{m}\right)$ in $L^{1 / 1} H_{0}^{1}$ is a Rademacher $l^{1}$ sequence in $L^{1}(\Pi)$, then also $\left(x_{m}\right)$ has this property in $L^{1} / H_{0}^{1}$. Previously, only a weaker subsequence version was known to be true.

Remarks. (1) With some additional work, one can replace $\delta^{2}$ by $\delta$ in (i) of Theorem 4.1. For what follows, this is however of no relevance.

(2) Assume $x_{1}, x_{2}, \ldots, x_{n}$ a sequence in $L^{1} / H_{0}^{1}$. It follows from the results of section 2 that there exist functions $f_{m} \in L^{1}(\Pi)$ such that

$$
q\left(f_{m}\right)=x_{m} \text { and } \int\left\|\sum \varepsilon_{m} f_{m}\right\|_{1} d \varepsilon \leqslant C \int\left\|\sum \varepsilon_{m} x_{m}\right\| d \varepsilon .
$$

Unfortunately this procedure does not provide a pointwise lifting, which we need to prove certain results. We don't know if minimum-norm lifting preserves the Rademacher means.

\section{Further results on projections and topological properties of $\boldsymbol{H}^{\infty}$ and duals}

In this section we present consequences of the result on minimum norm lifting (Theorem 0.3. Theorem 4.1), proved in section 4, for finite rank projections in $H^{\infty}$ and weakly compact subsets of $\left(H^{\infty}\right)^{*}$.

\subsection{Finite dimensional complemented subspaces of $\boldsymbol{H}^{\infty}$}

As a first application of Theorem 0.3, we show that complemented subspaces of the disc algebra contain large $l^{\infty}$-subspaces. This completes results obtained in section 2 .

THEOREM 5.1. Given $M<\infty$, there exist $\delta=\delta(M)$ and $\lambda=\lambda(M)$ such that each $n$ dimensional norm-M complemented subspace $E$ of $H^{\infty}$ has a subspace $F$ satisfying

(i) $\operatorname{dim} F=m \geqslant \delta n$

(ii) $d\left(F, l_{m}^{\infty}\right) \leqslant \lambda$.

The reader will find the proof of follwowing general lemma in [10] (p. 116).

LEMMA 5.2. Given $\varepsilon>0$, there exists $\delta=\delta(\varepsilon)$ such that if $x_{1}, x_{2}, \ldots, x_{n}$ are norm-1 vectors in a Banach space $X$ and $x_{1}^{*}, x_{2}^{*}, \ldots, x_{n}^{*}$ norm- 1 in $X^{*}$ satisfying

(i) $\left\langle x_{k}, x_{k}^{*}\right\rangle=\varepsilon \quad(1 \leqslant k \leqslant n)$ 
(ii) $\left\|\Sigma \varepsilon_{k} x_{k}^{*}\right\| \leqslant 1$ for all $\varepsilon_{k}= \pm 1$,

then there exists a subset $D$ of $\{1,2, \ldots, n\}$ with

(iii) $\operatorname{card}(D) \geqslant \delta n$

(iv) $(\varepsilon / 2) \max _{D}\left|a_{k}\right| \leqslant\left\|\Sigma_{k \in D} a_{k} x_{k}^{*}\right\| \leqslant 2 \max _{D}\left|a_{k}\right|$.

In particular, $\left[x_{1}^{*}, x_{2}^{*}, \ldots, x_{n}^{*}\right]$ contains $l^{\infty}$ s of proportional dimension.

(The space $X$ in Lemma 5.2 can be assumed real or complex.)

In fact, Theorem 5.1 extends a result which was previously obtained for finite dimensional $P_{\lambda}$-spaces (see [12]). Also here, we use the same approach. Recall that $\pi_{2}^{(n)}$ denotes the 2-summing norm computed with respect to $n$ vectors.

Proof of Theorem 5.1. Denote $i: E \rightarrow H^{\infty}$ the injection and $P: H^{\infty} \rightarrow E$ the projection. It is clear, using local reflexivity, that we can assume $P^{*}$ ranging in $L^{1} / H_{0}^{1}$.

By the ideal property and N. Tomczak-Jaegerman's result [56], one has

$$
M \sqrt{2} \pi_{2}^{(n)}(i) \geqslant\|P\| \pi_{2}(i) \geqslant \pi_{2}\left(\operatorname{Id}_{E}\right)=\sqrt{n} .
$$

Therefore, one can find vectors $\varphi_{1}, \varphi_{2}, \ldots, \varphi_{n}$ in $E$ satisfying

$$
\left(\sum\left\|\varphi_{k}\right\|^{2}\right)^{1 / 2} \geqslant \frac{\sqrt{n}}{\sqrt{2} M} \sup \left(\sum\left|\left\langle\varphi_{k}, x\right\rangle\right|^{2}\right)^{1 / 2},
$$

where the sup is taken over all norm-1 vectors $x$ in $L^{1 /} H_{0}^{1}$. Notice that this supremum equals

$$
\left\|\left(\sum\left|\varphi_{k}\right|^{2}\right)^{1 / 2}\right\|_{\infty}
$$

Take norm-1 vectors $x_{1}, x_{2}, \ldots, x_{n}$ in $E^{*}$ such that

$$
\left\|\varphi_{k}\right\|=\left\langle\varphi_{k}, x_{k}\right\rangle \quad(1 \leqslant k \leqslant n)
$$

Denote $f_{k} \in L^{1}(\Pi)$ the minimum-norm lifting of $P^{*}\left(x_{k}\right) \in L^{1} / H_{0}^{1}$. There is a sequence $\sigma_{1}, \sigma_{2}, \ldots, \sigma_{n}$ of positive scalars satisfying

$$
\sum \sigma_{k}^{2}=1 \text { and } \sum \sigma_{k}\left\langle\varphi_{k}, f_{k}\right\rangle=\left(\sum\left\|\varphi_{k}\right\|^{2}\right)^{1 / 2}
$$


Hence

$$
\int\left(\sum\left|\varphi_{k}\right|^{2}\right)^{1 / 2}\left(\sum \sigma_{k}^{2}\left|f_{k}\right|^{2}\right)^{1 / 2} \geqslant \frac{\sqrt{n}}{\sqrt{2} M}\left\|\left(\sum\left|\varphi_{k}\right|^{2}\right)^{1 / 2}\right\|_{\infty}
$$

and thus

$$
\int\left(\max \left|f_{k}\right|\right)^{1 / 2}\left(\sum \sigma_{k}^{2}\left|f_{k}\right|\right)^{1 / 2} \geqslant \frac{\sqrt{n}}{\sqrt{2} M}
$$

from where

$$
\max _{1 \leqslant k \leqslant n}\left|f_{k}\right| \geqslant \frac{n}{2 M^{2}}
$$

Thus there is a set $J \subset\{1,2, \ldots, n\}$, card $(J) \geqslant n / 4 M^{2}$ and disjoint subsets $\left(A_{k}\right)_{k \in J}$ of $\Pi$ with

$$
\int_{A_{k}}\left|f_{k}\right| \geqslant \frac{1}{4 M^{2}} \text { for } k \in J
$$

Thus Theorem 0.4 applies and yields $H^{\infty}$-functions $\left(g_{k}\right)_{k \in J}$ fulfilling

$$
\sum_{J}\left|g_{k}\right| \leqslant 1 \quad \text { and }\left\langle f_{k}, g_{k}\right\rangle=\delta_{1} \quad(k \in J)
$$

Defining $x_{k}^{*}=P\left(g_{k}\right)$, we get

$$
\left\|\sum_{J} \varepsilon_{k} x_{k}^{*}\right\|_{\infty} \leqslant\|P\| \text { for }\left|\varepsilon_{k}\right|=1
$$

and

$$
\left\langle x_{k}, x_{k}^{*}\right\rangle=\delta_{1} \text { for } k \in J
$$

So Lemma 5.2 concludes the proof.

\subsection{Weakly completeness and Dunford-Pettis property}

Our next purpose is to extend weakly completeness and Dunford-Pettis property of $L^{1} / H_{0}^{1}$ (cf. [44], chapter VII) to the odd duals of $H^{\infty}$.

There are different ways to proceed. We will use here the ultraproduct technique. 
Recall that if $X$ is a Banach space, $I$ a set and $\mathscr{U}$ an ultra-filter on $I$, the ultra-power $X_{\mathscr{U}}$ is defined as the quotient of the $l^{\infty}$-sum $\left(\Sigma_{I} X\right)_{\infty}$ by its subspace $N_{\mathscr{U}}=\left\{\left(x_{i}\right)_{i \in I} ; \lim _{\mathscr{U}}\left\|x_{i}\right\|=0\right\}$. We recall also that $X^{* *}$ is isometric to a 1-complemented subspace of some ultra-power of $X$. The reader is referred to [55] for more details.

THEOREM 5.3. Any ultrapower of $L^{1 /} H_{0}^{1}$ is weakly complete and has DPP.

So, by previous observations

COROLLARY 5.4. (i) $H^{\infty}$ and its duals are DP-spaces.

(ii) The odd duals of $H^{\infty}$ are weakly complete.

The reader is also referred to [9] for further related results (see remarks below).

If $\left(L^{1} / H_{0}^{1}\right)_{u}$ is an ultrapower of $L^{1} / H_{0}^{1}$ and $\left(L^{1}\right)_{u}$ the corresponding ultrapower of $L^{1}(\Pi)$, we can consider the natural extensions of $q$ and $\sigma$ to these ultrapowers

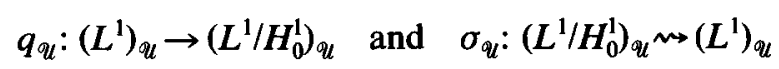

It is then clear that $\sigma_{\mathscr{U}}$ is a minimum-norm lifting of $q_{\mathscr{U}}$.

Since any ultra-power $\left(L^{1}\right)_{u}$ of $L^{1}$ is an $L^{1}(\mu)$-space and hence satisfies the properties considered in Theorem 5.3, it will suffice to prove that $\sigma_{\mathscr{U}}$ maps WCC sets onto WCC sets. Thus (cf. [16], [51]).

Proposition 5.5. Assume $\left(\xi_{k}\right)$ a sequence in $\left(L^{1} / H_{0}^{1}\right)_{{ }_{u}}$ such that the sequence of liftings $\sigma_{U}\left(\xi_{k}\right)$ is equivalent to the usual $l^{1}$-basis in $\left(L^{1}\right)_{\varkappa}$. Then $\left(\xi_{k}\right)$ has a subsequence $\left(\xi_{k}^{\prime}\right)$ which is equivalent to the $l^{1}$-basis in $\left(L^{1} / H_{0}^{1}\right)_{u}$.

Proof. We show that $\left(\xi_{k}\right)$ is an $l^{1}$-sequence in the Rademacher sense.

The argument is routine.

Denote for convenience $\xi_{k}=\sigma_{\sigma_{u}}\left(\xi_{k}\right)$. Thus

$$
\zeta_{k}(i)=\sigma\left(\xi_{k}(i)\right) \text { for each } i \in I
$$

where

$$
\left(\xi_{k}(i)\right)_{i \in I} \text { represents } \xi_{k} \text { in }\left(L^{1} / H_{0}^{1}\right)_{U} .
$$

Assume $\delta>0$ such that

$$
\delta \sum\left|c_{k}\right| \leqslant\left\|\sum^{\prime} c_{k} \zeta_{k}\right\| \leqslant \delta^{-1} \sum\left|c_{k}\right| .
$$


For fixed $\lambda>1$, one can choose for each $n=1,2, \ldots$ some element $U \in \mathcal{U}$ satisfying

$$
\lambda^{-1}\left\|\sum c_{k} \zeta_{k}\right\| \leqslant\left\|\sum_{k=1}^{n} c_{k} \zeta_{k}(i)\right\| \leqslant \lambda\left\|\sum c_{k} \zeta_{k}\right\|
$$

for all scalars $c_{1}, c_{2}, \ldots, c_{n}$, whenever $i \in U$. Hence. for $i \in U$

$$
\int\left\|\sum_{k=1}^{n} \varepsilon_{k} c_{k} \sigma\left(\xi_{k}(i)\right)\right\| d \varepsilon \geqslant \lambda^{-2} \delta^{2} \sum\left|c_{k}\right|\left\|\xi_{k}(i)\right\|
$$

and we see that for an appropriate $\delta_{1}>0$, also

$$
\int\left\|\sum_{k=1}^{n} \varepsilon_{k} c_{k} \xi_{k}(i)\right\| d \varepsilon \geqslant \delta_{1} \sum\left|c_{k}\right|\left\|\xi_{k}(i)\right\| .
$$

Therefore

$$
\int\left\|\sum_{k=1}^{n} \varepsilon_{k} c_{k} \xi_{k}\right\| d \varepsilon \geqslant \delta_{1} \sum\left|c_{k}\right|\left\|\xi_{k}\right\|
$$

completing the proof.

A slight modification of the preceding argument shows in fact that for fixed $\xi \in\left(L^{1} / H_{0}^{1}\right)_{U}$ the set $\left\{\zeta \in\left(L^{1}\right)_{\tau_{u}} ; q_{k}(\zeta)=\xi,\|\zeta\|=\|\xi\|\right\}$ is weakly compact in $\left(L^{1}\right)_{\varkappa_{U}}$. On the other hand, one verifies easily that an element of $\left(H^{\infty}\right)^{*}$ has not necessarily a unique norm-preserving extension to an element of $\left(L^{\infty}\right)^{*}$. However

COROLlaRY 5.6. For each $\xi \in\left(H^{\infty}\right)^{*}$, the set $\left\{\zeta \in\left(L^{\infty}\right)^{*} ; q^{* *}(\zeta)=\xi,\|\xi\|=\|\xi\|\right\}$ is weakly compact.

Proof. The ultraproduct representation allows to embed $\left(H^{\infty}\right)^{*},\left(L^{\infty}\right)^{*}$ in respective ultrapowers $\left(L^{1} / H_{0}^{1}\right)_{U},\left(L^{1}\right)_{\mathscr{U}}$ for the same ultrafilter $U$ and so that moreover the scheme

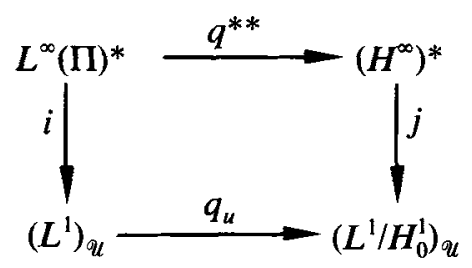


is commutative. From the preceding observation, it follows that $\left\{i(\zeta) ; q^{* *}(\zeta)=\xi\right.$, $\|\xi\|=\|\xi\|\}$ is a weakly compact subset of $\left(L^{1}\right)_{u}$. Hence the assertion follows.

Remark. Using fully Theorem 4.1, the reasoning in Proposition 5.5 shows that any $l^{1}$-sequence in $\left(L^{1 /} / H_{0}^{1}\right)_{\ell}$ has a subsequence with linear span complemented in $\left(L^{1} / H_{0}^{1}\right)_{u}$. In [9], it is shown that $l^{1}$-sequences in $\left(H^{\infty}\right)^{*}$ have complemented subsequences with biorthogonal functionals lying in $H^{\infty}$. As a consequence, any non-weakly compact operator on $H^{\infty}$ fixes an $l^{\infty}$-copy and, in particular, $l^{\infty}$ embeds in each infinite dimensional complemented subspace of $H^{\infty}$. The proof of this fact is rather long and technical and will not be presented here.

\section{Further remarks}

(1) Several of the previous results go through for closed subalgebra's $B$ of $L^{\infty}(\Pi)$ containing $H^{\infty}$. Indeed, by the Douglas property, each finite subset of $B$ can be approximated by elements of $\bar{b} \cdot H^{\infty}$, for some Blaschke product $b$ with $\bar{b} \in B$ (see [39] or [20], p. 378).

For instance, this fact allows to prove that $B\left(B, l^{l}\right)=\Pi_{2}\left(B, l^{l}\right)$ and $B^{*}$ is of cotype 2 .

(2) The Grothendieck property of $A^{*}$ can be rephrased in terms of tensor-algebra's (see [22], p. 308 for definitions). It is equivalent to the fact that the projective tensor algebra $A \widehat{\otimes} A$ is closed in $C(\Pi) \widehat{\otimes} C(\Pi)$. Indeed, the dual statement means that given $T \in B\left(A, A^{*}\right)$, there exists $\tilde{T} \in B\left(C, C^{*}\right)$ such that $T=j^{*} \bar{T} j$, where $j$ is the embedding. This solves a question raised by $\mathrm{N}$. Varopoulos.

(3) The results of section 2 were used in a more recent construction, due to G. Pisier, of a Banach space $X$ for which the two tensor-products $X \widehat{\otimes} X$ and $X \widehat{\widehat{\otimes}} X$ coincide (see [50]). More precisely, one uses the existence of an embedding $T$ of $l^{2}$ in $L^{1} / H^{1}$ such that $T^{*}$ is 1 -summing and the liftability of finite sequences in $L^{1} / H^{1}$ to $L^{1}(\Pi)$, preserving the Rademacher mean.

(4) Besides the spaces $C_{\Lambda}$ considered at the end of section 2 and remark 1, we don't know of other extensions of our results. Natural candidates are the polydiscalgebra's, the ball-algebra's and the spaces of continuously differentiable functions, on the Banach-space structure of which not much seems to be known.

(5) Taking in Theorem $0.4 f_{m}=P_{z_{m}}$, i.e. the Poisson kernels of points $z_{m}$ in $D$; one obtain essentially J. Garnett's theorem that the notions of harmonically interpolating sequence and interpolating sequence coincide (see [21]). The author proved previously Theorem 4.1 by a constructive argument, in particular not relying on factorization. 
(6) Finally, let us notice that apparently no example is known of a subset $\Lambda$ of $\mathbf{Z}$ for which $C_{\mathrm{A}}$ fails Dunford-Pettis property.

Added in proof. More recently, it was shown by the author that bounded linear operators from $H^{\infty}$ into a Banach space with cotype property factor through $L^{\infty}$, also without approximation hypothesis. This fact solves the problem related to Corollary 2.8 , in section 2 of the paper.

\section{References}

[1] BeAuZAmy, E. \& MAUREY, B., Opérateurs de convolution $r$-sommants sur un groupe compacte abélien. C. R. Acad. Sci. Paris, 277 (1973), 113-115.

[2] BERGH, J. \& LÖFSTRÖM, J., Interpolation spaces, an introduction. Grundlehren der mathematischen Wissenschaften 223, Springer Verlag.

[3] Bockariov, S. V., Existence of a basis in the space of analytic functions and some properties of the Franklin system. Mat. Sb., 24 (1974), 1-16.

[4] Bourgain, J., Propriétés de relèvement et projections dans les espaces $L^{1 /} H_{0}^{1}$ et $H^{\infty}$. C. $R$. Acad. Sci. Paris Sér. A, 291 (1980), 607-609.

[5] - Sur les projections dans $H^{\infty}$ et la propriété de Grothendieck. C.R. Acad. Sci. Paris Sér. I, 293 (1981), 47-49.

[6] - Opérateurs sommants sur l'algèbre du disque. C. R. Acad. Sci. Paris Sér I, 293 (1981), $677-680$.

[7] - Nouvelles propriétés des espaces $L^{1} / H_{0}^{1}$ et $H^{\infty}$. Sém. d'Anal. Fonct. 1980-81, Exp. III, Ecole Polytechnique.

[8] - A theorem on interpolating sequences. Simon Stevin, 57 (1983), 145-155.

[9] $-H^{\infty}$ is a Grothendieck space. Studia Math., 75 (1982), 193-226.

$[10]$ - New classes of $\mathscr{L}^{p}$ spaces. Springer Lecture Notes in Mathematics, 889.

[11] - New Banach space properties of the disc algebra and $H^{\infty}$. Proc. Special Year in Analysis, University of Connecticut. To appear.

[12] - A remark on finite dimensional $\boldsymbol{P}_{\lambda}$-spaces. Studia Math., 72 (1981), 87-91.

[13] Bourgain, J. \& Pelczynski, A., In preparation.

[14] Carleson, L., An interpolation problem for bounded analytic functions. Amer. J. Math., 80 (1958), 921-930.

[15] Chaumat, J., Une généralisation d'un théorème de Dunford-Pettis. Université Paris XI, U. E. R. Mathématique. Preprint no 85, 1974.

[16] Dor, L., On sequences spanning a complex $l^{1}$ space. Proc. Amer. Math. Soc., 47 (1975), 515-516.

[17] - On projections in $L^{1}$. Ann. of Math., 102 (1975), 463-474.

[18] Duren, P., Theory of $H^{p}$ spaces. Academic Press, New York and London, 1970.

[19] Figiel, T. \& Pisier, G., Séries aléatoires dans les espaces uniformément convexes ou uniformément lisses. C. R. Acad. Sci. Paris Sér. A, 279 (1974), 611-614.

[20] Garnett, J., Bounded analytic functions. Academic Press, 1981.

[21] - Interpolating sequences for bounded harmonic functions. Indiana Univ. Math. J., 21 (1971), 187-192.

[22] Graham, C. C. \& McGehee, O. C., Essays in commutative harmonic analysis. Grundlehren der mathematischen Wissenschaften 238, Springer Verlag. 
[23] Grothendieck, A., Résumé de la théorie métrique des produits tensoriels topologiques. Bol. Soc. Matem., Sao Paulo, 8 (1956), 1-79.

[24] - Produits tensoriels topologiques et espaces nucléaires. Memoirs A. M. S., 16 (1955).

[25] Havin, V. P., Weak sequential completeness of the spaces $L^{1} / H_{0}^{1}$. Vestnik Leningrad Univ., 13 (1973), 77-81.

[26] - Spaces $H^{\infty}$ and $L^{1} / H_{0}^{1}$. Isslied. po Lin. Operatoram i Tieor. Funkeii IV, Zapiski Nowc Sem. LOMI, 29 (1974), 120-148.

[27] Hoffman, K., Banach spaces of analytic functions. Prentice Hall, Englewood Cliffs, N. Y. 1962.

[28] JoNES, P., $L^{\infty}$-estimates for the $\partial$-problem in a half-plane. Acta Math., 150 (1983), 137-152.

[29] Kisliakov, S. V., On the conditions of Dunford-Pettis, Pelczynski and Grothendieck. Dokl. Akad. Nauk SSSR, 225 (1975), 152-155.

[30] - On reflexive subspaces of $C_{A}^{*}$. Funkcional, Anal. i Priložen, 13 (1979), 21-30.

[31] - On spaces with "small" annihiliators. Zap Naučn Sem. Leningrad Otdel Mat. Inst. Steklov (LOMI), 65 (1976), 192-195.

[32] - What is needed for a 0 -absolutely summing operator to be nuclear. Preprint.

[33] - Two remarks on the equality $\Pi_{p}(X, \cdot)=I_{p}(X, \cdot)$. Isslied. po Lin. Operatoram $i$ Tieor Funkeii XI, Zapiski Nowo Sem. LOMI, 113 (1981), 135-148.

[34] Krivine, J. L., Théorèmes de factorisation dans les espaces réticulés. Sém. MaureySchwartz 1973-74, Exp. 22-33.

[35] Kwapien, S. \& Pelczynski, A., Absolutely summing operators and translation invariant spaces of functions on compact abelian groups. Math. Nachr., 94 (1980), 303-340.

[36] Lindenstrauss, J. \& Tzafriri, L., Classical Banach spaces. Lecture Notes in Mathematics 338, Springer Verlag, 1973.

[37] - Classical Banach spaces I, Ergebnisse der Mathematik 92, Springer Verlag, 1977.

[38] - Classical Banach spaces II. Ergebnisse der Mathematik 97, Springer Verlag, 1979.

[39] Marshall, D., Subalgebras of $L^{\infty}$ containing $H^{\infty}$. Acta Math., 137 (1976), 81-98.

[40] Maurey, B., Un théorème de prolongement. C. R. Acad. Sci. Paris Sér A-B, 279 (1974), 329-332.

[41] — Théorème de factorisation pour les opérateurs linéaires à valeurs dans les espaces $L^{p}$. Astérisque 11 (1979).

[42] Maurey, B. \& Pisier, G., Séries de variables aléatoires vectorielles indépendantes et propriétés géométriques des espaces de Banach. Studia Math., 58 (1976), 45-90.

[43] Maurey, B., Une nouvelle démonstration d'un théorème de Grothendieck. Seminaire Maurey-Schwartz 1973-74, Exp. 22.

[44] Pelczynski, A., Banach spaces of analytic functions and absolutely summing operators. Conf. board of math., SC. Regional Conf. Ser. in Math. no 30 (1976).

[45] - Absolutely summing operators from the disc algebra. Isslied. po Lin. Operatoram $i$ Tieor. Funkeii, Zapiski Nowc Sem. LOMI, 81 (1978), 12-14.

[46] - Four lectures on translation invariant Banach spaces and Banach spaces of smooth and analytic functions. Séminarie d'Initiation à l'Analyse, $18^{\mathrm{e}}$ année (1978). Publ. Math. Univ. Pierre et Marie Curie, no 2.

[47] Pietch, A., Operator ideals. VEB Deutscher Verlag der Wissenschaften, Berlin 1978.

[48] Pisier, G., Une nouvelle classe d'espaces de Banach vérifiant le théorème de Grothendieck. Ann. Inst. Fourier, 28 (1978), 69-90.

[49] - Une propriété de stabilité de la classe des espaces se contenant par $l^{1}$. C. R. Acad. Sci. Paris. Sér. A-B, 286 (1978), 747-749.

[50] - Counterexamples to a conjecture of Grothendieck. Preprint.

[51] Rosenthal, H. P., A characterization of Banach spaces containing $l^{1}$. Proc. Nat. Acad. Sci. U.S.A., 71 (1974), 2411-2413. 
[52] SCHWARTZ, L., Geometry and probability in Banach spaces. Springer Lecture notes in mathematics $852,1981$.

[53] Stein, E., Singular integrals and differentiability properties of functions. Princeton Univ. Press, Princeton, 1970.

[54] STEIN, E. \& WeISS, G., Introduction to Fourier analysis on euclidean space. Princeton Academic Press, Princeton, 1971.

[55] Stern, J., Propriétés locales et ultrapuissances d'espaces de Banach. Seminaire Maurey. Schwartz 1974-75, Exp. VII, VIII, Ecole Polytechnique Paris.

[56] TOMCZAK-JAEgermanN, N., Computing 2-summing norm with few vectors. Ark. Mat., 17 (1979), 173-177.

[57] WoJTASzCZYK, P., On projections in spaces of bounded analytic functions with applications. Studia Math., 65 (1979), 147-173.

[58] - Decompositions of $H^{p}$ spaces. Duke Math. J., 46 (1979), 635-644.

[59] - Finite dimensional operators on spaces of analytic functions. Isslied po Lin. Operatoram i Tieor. Funkeii, Zapiski Nowo Sem. LOMI, 81 (1978), 18-19.

[60] ZipPIN, M., The finite-dimensional $P_{\lambda}$ spaces with small $\lambda$. Israel J. Math., 39 (1981), 359-364.

Received February 18, 1982

Received in revised form October 5, 1982 